\title{
Physical parameters of 15 intermediate-age LMC clusters from modelling of HST colour-magnitude diagrams
}

\author{
L. O. Kerber ${ }^{1}$, B. X. Santiago ${ }^{2}$, and E. Brocato ${ }^{3}$ \\ 1 Universidade de São Paulo, Rua do Matão 1226, Cidade Universitária, São Paulo 05508-900, SP, Brazil \\ e-mail: kerber@astro.iag.usp.br \\ 2 Universidade Federal do Rio Grande do Sul, IF, CP 15051, Porto Alegre 91501-970, RS, Brazil \\ 3 INAF - Osservatorio Astronomico di Collurania, via M. Maggini, 64100 Teramo, Italy
}

Received 27 July 2006 / Accepted 3 October 2006

\begin{abstract}
Aims. We analyzed HST/WFPC2 colour-magnitude diagrams (CMDs) of 15 populous Large Magellanic Cloud (LMC) stellar clusters with ages between $\sim 0.3 \mathrm{Gyr}$ and $\sim 3 \mathrm{Gyr}$. These $(V, B-V)$ CMDs are photometrically homogeneous and typically reach $V \sim 22$. Accurate and self-consistent physical parameters (age, metallicity, distance modulus and reddening) were extracted for each cluster by comparing the observed CMDs with synthetic ones.

Methods. These determinations involved simultaneous statistical comparisons of the main-sequence fiducial line and the red clump position, offering objective and robust criteria to determine the best models. The models explored a regular grid in the parameter space covered by previous results found in the literature. Control experiments were used to test our approach and to quantify formal uncertainties.

Results. In general, the best models show a satisfactory fit to the data, constraining well the physical parameters of each cluster. The age-metallicity relation derived by us presents a lower spread than similar results found in the literature for the same clusters. Our results are in accordance with the published ages for the oldest clusters, but reveal a possible underestimation of ages by previous authors for the youngest clusters. Our metallicity results in general agree with the ones based on spectroscopy of giant stars and with recent works involving CMD analyses. The derived distance moduli implied by the most reliable solutions, correlate with the reddening values, as expected from the non-negligible three-dimensional distribution of the clusters within the LMC.

Conclusions. The inferred spatial distribution for these clusters is roughly aligned with the LMC disk, being also more scattered than recent numerical predictions, indicating that they were not formed in the LMC disk. The set of ages and metallicities homogeneously derived here can be used to calibrate integrated light studies applied to distant galaxies.
\end{abstract}

Key words. galaxies: star clusters - Magellanic Clouds - Hertzsprung-Russell (HR) and C-M diagrams

\section{Introduction}

The Large Magellanic Cloud (LMC) is a useful ensemble of stars and stellar systems, since it is a galaxy with remarkably distinct characteristics when compared with to Galaxy, while its distance is close enough so that its stellar content is well resolved (Olszewski et al. 1996; Westerlund 1997). This rich information imprinted in the LMC includes its large system of more than 1800 identified stellar clusters (Bica et al. 1999). Some rich LMC clusters may be as old as Milky Way globular clusters; many others have ages similar to those inferred for the open clusters in the disk of our Galaxy, but are generally richer and more metal-poor than these are. Therefore, studies of individual LMC clusters, as well as of its entire cluster system, have lead to valuable contributions to the understanding of how clusters, and the stars within them, form and evolve.

Many examples can be found in the recent literature that illustrate this promising field. With respect to the impact on stellar evolution theory, evolutionary tracks and isochrones of young and subsolar metallicity stars are being continuously tested, resulting in stimulating discussion about the efficiency of the convective overshooting process (Brocato et al. 2003; Gallart et al. 2003). The spatial variation of the stellar luminosity and mass functions observed in clusters has helped us better understand the mass segregation effect (de Grijs et al. 2002; Gouliermis et al. 2004; Kerber \& Santiago 2006) and has thus contributed to the discussion about the IMF universality. In terms of the cluster systems, the lack of populous LMC clusters with ages between 4 and $10 \mathrm{Gyr}$ (the so-called "age gap"; the only known exception is ESO 121-SC03), also imprinted on the cluster agemetallicity relation (AMR) (Olszewski et al. 1991; Bica et al. 1998; Geisler et al. 1997), was recently reproduced by numerical $N$-body simulations with realistic conditions for the clusters formation that take into account the interaction between LMC, the Small Magellanic Cloud (SMC) and the Galaxy (Bekki et al. 2004; Bekki \& Chiba 2005).

LMC clusters also provide a decisive contribution to the calibration of models describing the integrated light (spectra and colours) of single stellar populations (SSP). These models in turn are crucial for the studies of distant unresolved stellar populations. Therefore, it is necessary that these models recover ages and metallicities of LMC clusters which are in agreement with those obtained by methods that rely on the analysis of resolved stars. Otherwise there is a serious risk that the interpretation of the stellar content of unresolved galaxies will be severely biased. Several recent works either fully or partially 
study the integrated light of LMC clusters: Leonardi \& Rose (2003), Santos \& Piatti (2004), Santos et al. (2006), de Grijs \& Anders (2006), McLaughlin \& van der Marel (2005), Beasley et al. (2002), Goudfrooij et al. (2006). The results of all these studies on the LMC clusters are founded on two observational pillars: the spectroscopy of individual red giants and the photometry of dense systems that make up colour-magnitude diagrams (CMDs). While the former provides metallicities, largely based on the calcium triplet lines, the latter yield ages, metallicities, distance modulus and reddening values.

Spectroscopic studies include those by Olszewski et al. (1991) (hereafter OSSH), which determined $[\mathrm{Fe} / \mathrm{H}]$ for $\sim 70$ LMC clusters, and Cole et al. (2005), which did the same for 373 LMC field stars. Recently, Geisler (2006) (see also Grocholski et al. 2006) showed new results for 29 clusters observed with the FORS2 instrument on the Very Large Telescope (VLT), where typically 8 red giants per clusters were used to constrain the metallicity of each cluster. This latter work, that exceeds the OSSH in quality but covers a lower number of clusters, is a good example of the successful application of multi-object spectroscopy (MOS) for LMC clusters.

As for CMD analysis, it has been used as a powerful tool to determine the physical parameters of stellar systems as well as to calibrate stellar evolution theory. CMDs based on Hubble Space Telescope (HST) or on $8 \mathrm{~m}$ class telescopes, coupled with detailed analysis techniques, have allowed accurate determinations of age and metallicities for LMC clusters (Kerber \& Santiago 2005; Bertelli et al. 2003; Woo et al. 2003) and star formation history (SFH) for neighbouring galaxies, including the LMC (Gallart et al. 1999; Dolphin 2002; Javiel et al. 2005). A common feature of the aforementioned works is that they combine synthetic CMDs (generated by numerical simulations) with statistical tools to discriminate the best models, constituting a testable and objective approach to recover the physical information from an observed CMD. This kind of study, if applied to a large number of clusters, should significantly improve the age determinations based on lower-resolution data (e.g., Elson \& Fall 1988; Girardi et al. 1995). For instance, Dirsch et al. (2000), Geisler et al. (2003), Piatti et al. (2003a,b) are examples of recent works that applied homogeneous analyses to CMDs taken from a sizable number of LMC clusters, although their data still come from ground-based small telescopes.

With this in mind we analyzed a sample of HST data taken with the Wide Field and Planetary Camera 2 (WFPC2) CMDs of populous LMC clusters published by Brocato et al. (2001). We selected the 15 intermediate-age clusters (IACs) in this sample that satisfy the following criteria: i) inferred age between $\sim 0.3$ to $\sim 3.5 \mathrm{Gyr}$; ii) CMDs that display a main-sequence (MS) stretching at least 1 mag below the turn-off (MSTO) point and with evidence of red clump (RC) stars.

These data are photometrically homogeneous and typically reach $V \sim 22$ for stars in the cluster's centre. The main goal of this work is to provide ages, metallicities, distance moduli and reddening values for each cluster in a self-consistent method based on a homogeneous and robust analysis. Therefore, these physical parameters can be very useful for the calibration of integrated light SSP models. Furthermore, the set of derived distance moduli for individual clusters offers a good opportunity to probe the three-dimensional distribution of the intermediate-age clusters within the LMC, adding new information and important constraints to the understanding of the stellar cluster formation in this neighbouring galaxy.

In the next section we present the observed CMDs and the cluster sample. The CMD modelling process is presented in
Sect. 3 while the model grid and the previous determinations found in the literature are shown in Sect. 4 . Section 5 is dedicated to the statistical tools that objectively discriminate among the best models. The results are presented and discussed in Sect. 6; we first discuss the results on a cluster-by-cluster basis, but also investigate the sample properties as a whole. The last section shows the conclusions and the summary.

\section{The data}

The data used in the present work were taken with the HST/WFPC2 for the following 15 rich intermediate-age LMC stellar clusters: NGC 1651, NGC 1718, NGC 1777, NGC 1831, NGC 1856, NGC 1868, NGC 2121, NGC 2155, NGC 2162, NGC 2173, NGC 2209, NGC 2213, NGC 2249, SL 506 and SL663. These data were selected from the HST archive and reduced by Brocato et al. (2001). Their original cluster sample has 21 LMC clusters and one SMC cluster, covering a wider range in age $(0.1 \lessgtr \tau \lesssim 13$ Gyr). For each cluster, snapshot images were obtained in the F450W $(\sim B)$ and F555W $(\sim V)$ filters, and photometrically transformed to the standard system. The HST archival images, data reduction process, procedures to calibrate the data and to evaluate the incompleteness are all detailed in Brocato et al. (2001).

The final $(V, B-V)$ CMDs from Brocato et al. (2001) for the 15 intermediate-age LMC clusters are presented in Fig. 1. These CMDs include only central stars, therefore reducing significantly the contamination by field LMC stars. We adopt a cut-off radius of $R \leq 2 R_{\mathrm{c}}$, where $R_{\mathrm{c}}$ is the core radius for all clusters, except NGC 2121. This latter is located in one of the most contaminated directions towards the LMC and has one of the faintest MS terminations; we thus selected inner stars $\left(R \leq 1 R_{\mathrm{c}}\right)$ to avoid field star contamination. These are the CMDs we used to extract the physical parameters of each cluster (see Sect. 6).

We notice two common features in the CMDs: the extended MS $(V \sim 22)$ and the prominent presence of stars in the heliumburning RC phase. In this figure they follow the same sequence as suggested by Brocato et al. (2001) to reveal the age effect in a LMC cluster. As the cluster becomes older, both the MS termination and the RC become less bright. However, this latter stalls at $V \sim 19.0$. Thus the $V_{\mathrm{MSTO}}-V_{\mathrm{RC}}$ magnitude difference increases for clusters older than $\sim 1 \mathrm{Gyr}$, as a consequence being a good age indicator (Geisler et al. 1997; Castellani et al. 2003). Additional features that can be seen in the CMDs are the red giant branch (RGB) and, for the older clusters, the sub-giant branch (SGB).

The on-sky distribution of our cluster sample is shown in Fig. 2, together with the 30 Dor position and the line of nodes of the LMC disk, as determined by Nikolaev et al. (2004). The clusters have distances to the optical centre of LMC bar typically between $\sim 3^{\circ}$ and $\sim 6^{\circ}$, being spread in every quadrant with respect to this centre, but preferentially located in the east side of the LMC.

\section{CMD modelling}

To model a CMD we follow the numerical approach similar to that used by several authors in previous studies of resolved stellar populations (Gallart et al. 1999; Dolphin 2002; Bertelli et al. 2003; Woo et al. 2003; Kerber \& Santiago 2005). We generate synthetic CMDs that reproduce as accurately as possible the main features found in the observed CMD, finding the best model CMDs based on statistical comparisons in the 


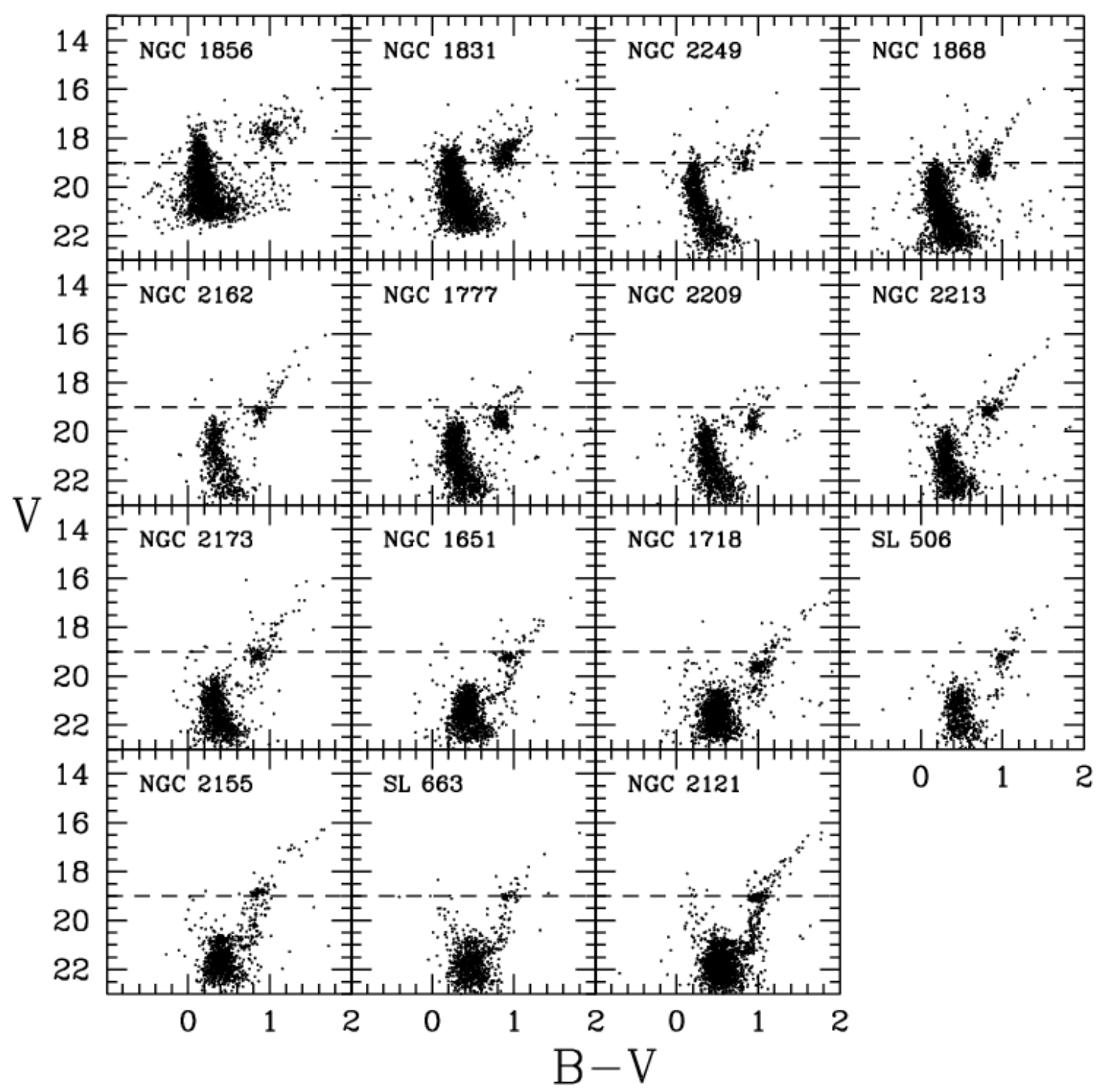

Fig. 1. CMDs for 15 intermediate-age LMC clusters by Brocato et al. (2001); only stars with $R \leq 2 R_{\mathrm{c}}\left(R \leq 1 R_{\mathrm{c}}\right.$ for NGC 2121) are shown. This sequence was suggested by those authors to reveal the "evolution" effect in the sample. The horizontal dashed line at $V=19.0$ corresponds to the MS end for a cluster with $\tau \sim 1.0 \mathrm{Gyr}$ at the LMC distance; it also roughly corresponds to the $V$ magnitude of RC stars in the oldest clusters.

CMD plane. The method is applicable to composed stellar populations (CSP), such as field stars in the Galaxy or in neighbouring galaxies, or to SSPs, as in the present case.

We thus modelled the CMDs of the LMC clusters, considering that each of them is an SSP, characterized by stars with the same age $(\tau)$ and metallicity $(Z)$ (described by a Padova isochrone, Girardi et al. 2002), placed at the same distance $((m-$ $\left.M)_{0}\right)$ and subjected to the same reddening $(E(B-V))$. These parameters uniquely define the position in the $(V, B-V)$ plane for single stars of a given mass. The number of stars in a given CMD position is fixed by the stellar mass distribution, more commonly referred to as the Present Day Mass Function (PDMF). It is parameterized here by a power law $(\mathrm{d} N / \mathrm{d} m \sim$ $m^{-\alpha}$ ). However, real observations suffer from photometric uncertainties and the effect of unresolved binaries (or blending of stars). The former effect is modelled using the photometric errors measured from the data. The effect of unresolved binaries is introduced by combining the fluxes of two synthetic stars in a fraction $\left(f_{\text {bin }}\right)$ of the CMD points. Only binaries whose secondary mass $\left(m_{2}\right)$ is at least $70 \%$ of the primary star mass $\left(m_{1}\right)$ contribute to $\left(f_{\text {bin }}\right)$, the masses being randomly selected according to a uniform mass ratio $\left(q=m_{2} / m_{1}\right)$ distribution. Therefore, $f_{\text {bin }}$ defined in this way incorporates only binaries whose CMD position is different from that of the primary stars alone.

To illustrate our CMD modelling process, in Fig. 3 we present examples of synthetic CMDs of rich LMC clusters. The CMDs are disposed in an age sequence, approximately covering the age range expected for the IACs from Brocato et al. (2001). As in Fig. 1, the effect of age in the CMDs is noticeable. The Padova isochrones used to generate the synthetic CMDs are also plotted in this figure. We deliberately adopt this set of stellar evolutionary models in this work because it presents the advantage of a thinner grid in age and metallicity than the others (see Sect. 4), being also widely used and tested, in general offering good fits to the data. Also the Padova isochrones adopt reasonable assumptions for convective overshooting, although Bertelli et al. (2003) found some evidence for a greater efficiency.

\section{Model grid and literature results}

For each cluster, we explored a grid of models covering the parameter space within reasonable limits. The ranges in age 


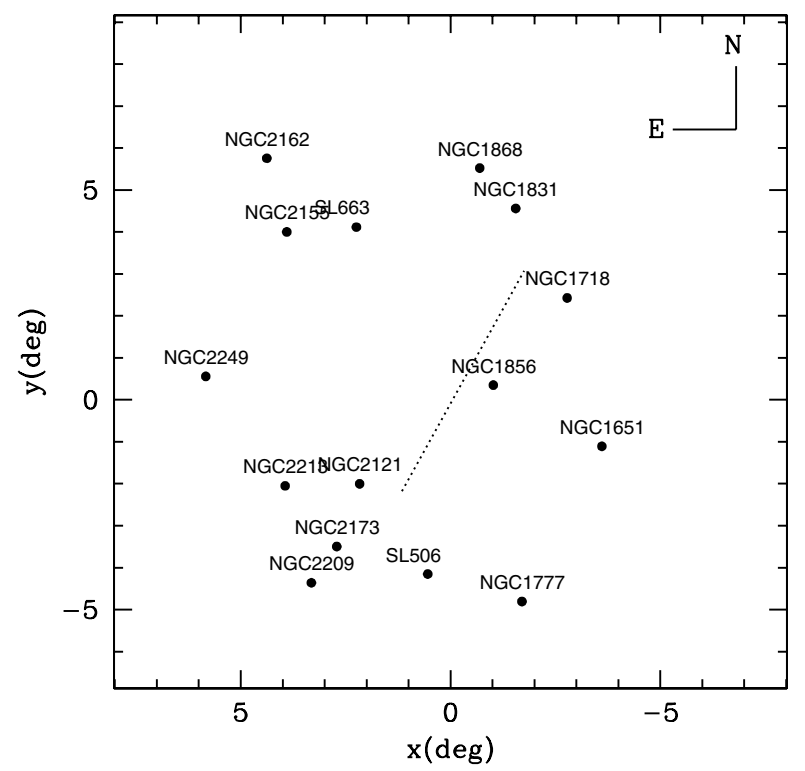

Fig. 2. Distribution on the sky of the LMC clusters (solid circles) analyzed in this work. The dotted line indicates the line of nodes of the LMC disk, as determined by Nikolaev et al. (2004), while the 30 Dor position is shown by an open circle. The positions are relative to the optical centre of the LMC bar, at RA $=05^{\mathrm{h}} \cdot 20^{\mathrm{m}} \cdot 56^{\mathrm{s}}$., Dec $=$ $-69^{\circ} 28^{\prime} 41^{\prime \prime}$ (J2000.0) (Bica et al. 1996).

and metallicity in the grid are consistent with the values compiled from the literature by Mackey \& Gilmore (2003) (hereafter MG03). These ranges are shown in the first row for each cluster in Table 1; they are meant to homogeneously cover the region in parameter space where the best models are expected to lie and therefore allow accurate age and metallicity determinations. All clusters have age results that come from CMD analyses, although carried out by different authors and with variable data quality. Among these, we quote Rich et al. (2001) based on HST data, and Geisler et al. (1997) who analysed homogeneous CMDs of 7 of our clusters. One of the pioneering works in LMC cluster age determination, Elson \& Fall (1988), completes the age list from MG03. The OSSH metallicity values, based on spectroscopy of red giants, were assumed. For those clusters not observed by OSSH, MG03 estimated a crude $[\mathrm{Fe} / \mathrm{H}]$ value based on the metallicities of clusters with similar ages, and therefore these estimates are only guidelines and should be used with caution. The ages compiled by MG03 are not necessarily consistent with the metallicities determined by OSSH, as the former results come from CMD analyses that also assumed an independent, and often different, metallicity value.

Since the publication of the MG03 compilation, some new ages and metallicities based on different techniques have been published. These results are also listed in Table 1, together with the ones from older works (Girardi et al. 1995; Girardi \& Bertelli 1998; Dirsch et al. 2000), which are still widely used. Also, we included the HST/CMDs analyses done by Sarajedini (1998) for the three oldest clusters in our sample, although he found solutions with overestimated ages and underestimated metallicities, as discussed and demonstrated by Rich et al. (2001). Recent works related to CMD analyses include (with the number of clusters in common with us and the telescope used shown in parenthesis): Bertelli et al. (2003) and Woo et al. (2003) (2, VLT); Piatti et al. (2003b) (3, CTIO 0.9m); Kerber \& Santiago (2005) (3, HST/WFPC2). Although less reliable than the CMD results, we also presented the ones obtained from the

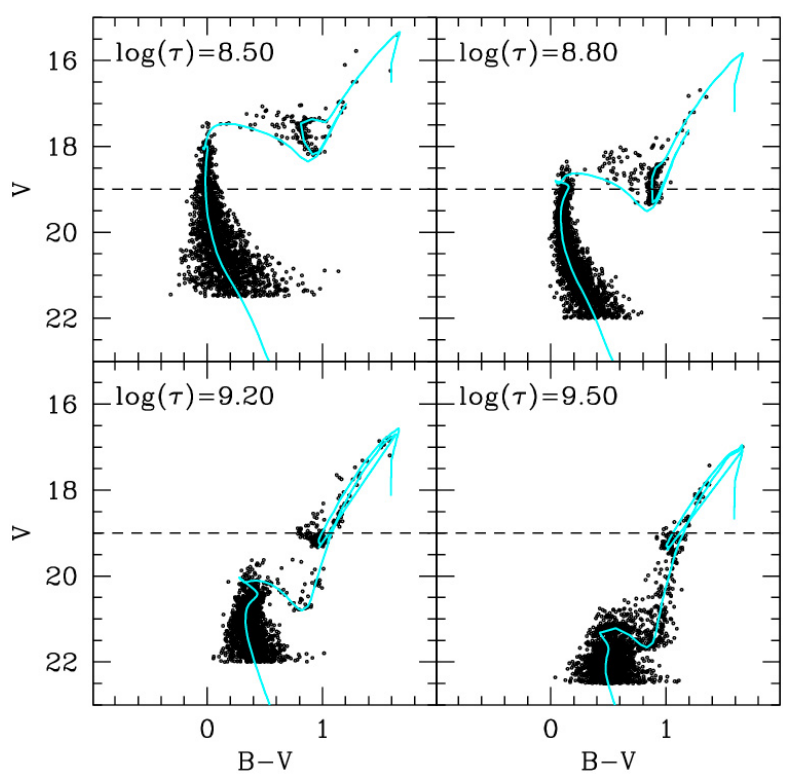

Fig. 3. Examples of synthetic CMDs of rich LMC clusters following a sequence in age, as indicated in each panel. The other physical parameters for these CMDs were fixed: $Z=0.008,(m-M)_{0}=18.50$, $E(B-V)=0.05, \alpha=2.00$ and $f_{\text {bin }}=30 \%$. The photometric uncertainties used in the models are consistent with the ones observed in the Brocato et al. (2001) sample. The horizontal dashed line at $V=19.0$ is a guideline for the MSTO and the RC position, as explained in Fig. 1.

analyses of integrated spectra done by Beasley et al. (2002) and Leonardi \& Rose (2003) because they offer a good opportunity to check the consistency of the derived age and metallicity that comes from this kind of technique.

To reduce the discreteness in the model grid we adopted the smallest age step published by Girardi et al. (2002), $\Delta \log (\tau / \mathrm{yr})=0.05$, and a thinner grid in $Z$ than the original one, kindly provided by L. Girardi using the TRILEGAL code (Girardi et al. 2005). This metallicity grid, illustrated in Fig. 4, is made up with $Z=0.0001,0.0004,0.002,0.004,0.006$, $0.008,0.012,0.016,0.019\left(Z_{\odot}\right), 0.024$ and 0.030 , where the new isochrones were obtained by interpolating between the original ones. To convert the $Z$ values to $[\mathrm{Fe} / \mathrm{H}]$, we assumed that $[\mathrm{Fe} / \mathrm{H}]=\log \left(Z / Z_{\odot}\right)$.

Since reddening and distance modulus were considered free parameters in the modelling, we explored ranges in the model grid that are compatible with the ones found for the LMC. The models span the range from $(m-M)_{0}=18.20(\sim 43.7 \mathrm{kpc})$ to $(m-M)_{0}=18.80(\sim 57.5 \mathrm{kpc})$ (with a step of 0.05) and from $E(B-V)=0.00$ to $E(B-V)=0.25$ (with a step of 0.01 ). While the first range is consistent with a spherical distribution of clusters with a radius of $\sim 9 \mathrm{kpc}$ (roughly $10 \mathrm{deg}$ on the sky) and centred at the canonical LMC distance $\left((m-M)_{0}=18.50\right)$, the second range is in accordance with the new reddening maps published for the LMC (Nikolaev et al. 2004; Zaritsky et al. 2004; Subramaniam 2005). Although in some cases these authors derived $E(B-V)$ values for directions close to the clusters, we preferred not to fix reddening for any cluster, since it can be located in the foreground or background relative to the bulk of the stars considered in these works.

The PDMF slope was fixed at $\alpha=2.00$, in agreement with the recent determinations for the central regions of LMC clusters (Kerber \& Santiago 2006). We adopted a typical value for the fraction of binaries of $f_{\text {bin }}=30 \%$, in accordance with the determination done by Elson et al. (1998) for inner parts of 
Table 1. Ages and metallicities summarized by Mackey \& Gilmore (2003) (first row for each cluster) and found in the literature for all clusters in our sample.

\begin{tabular}{|c|c|c|c|c|c|c|c|c|c|}
\hline Cluster & $\overline{l o g}(\tau / \mathrm{yr})$ & Ref. & {$[\overline{\mathrm{Fe}} / \mathrm{H}]$} & Ref. & Cluster & $\log (\tau / \mathrm{yr})$ & Ref. & {$[\mathrm{Fe} / \mathrm{H}]$} & Ref. \\
\hline \multirow[t]{4}{*}{ NGC 1651} & $9.30_{-0.10}^{+0.08}$ & 5 & $-0.37 \pm 0.20$ & 12 & NGC 2162 & $9.11_{-0.16}^{+0.12}$ & 5 & $-0.23 \pm 0.20$ & 12 \\
\hline & $9.34 \pm 0.08$ & 8 & $-0.82 \pm 0.44$ & 8 & & $9.32 \pm 0.06$ & 8 & $-0.90 \pm 0.03$ & 8 \\
\hline & $9.26 \pm 0.08$ & 15 & $-0.07 \pm 0.10$ & 15 & & $8.95 \pm 0.10$ & 6 & & \\
\hline & 9.40 to 9.10 & 3 & -0.63 to -0.45 & 3 & NGC 2173 & $9.33_{-0.09}^{+0.07}$ & 5 & $-0.24 \pm 0.20$ & 12 \\
\hline \multirow[t]{3}{*}{ NGC 1718} & $9.30 \pm 0.30$ & 4 & $\sim-0.42$ & 10 & & $9.19 \pm 0.04$ & 2 & -1.05 to -0.80 & 2 \\
\hline & $9.30_{-0.14}^{+0.16}$ & 1 & $-0.98_{-0.30}^{+0.29}$ & 1 & & $9.62 \pm 0.05$ & 8 & $-1.38 \pm 0.08$ & 8 \\
\hline & $9.69_{-0.07}^{+0.05}$ & 1 & $-1.12_{-0.22}^{+0.18}$ & 1 & & $9.18 \pm 0.08$ & 16 & $\sim-0.7$ & 16 \\
\hline \multirow[t]{2}{*}{ NGC 1777} & $9.08_{-0.18}^{+0.12}$ & 5 & $-0.35 \pm 0.20$ & 12 & & $9.06 \pm 0.10$ & 6 & & \\
\hline & $9.01 \pm 0.05$ & 8 & $-0.39 \pm 0.01$ & 8 & NGC 2209 & $8.98_{-0.24}^{+0.15}$ & 5 & $\sim-0.47$ & 10 \\
\hline \multirow[t]{4}{*}{ NGC 1831} & $8.50 \pm 0.30$ & 4 & $+0.01 \pm 0.20$ & 12 & & $\sim 9.18$ & 11 & & \\
\hline & $8.70 \pm 0.03$ & 9 & $-0.20 \pm 0.10$ & 9 & & $8.96 \pm 0.10$ & 6 & & \\
\hline & $8.70 \pm 0.14$ & 8 & $-0.65 \pm 0.02$ & 8 & NGC 2213 & $9.20_{-0.12}^{+0.10}$ & 5 & $-0.01 \pm 0.20$ & 12 \\
\hline & $8.60 \pm 0.10$ & 6 & & & & $9.32 \pm 0.02$ & 8 & $-0.88 \pm 0.06$ & 8 \\
\hline \multirow{4}{*}{ NGC 1856} & $8.12 \pm 0.30$ & 4 & $\sim-0.52$ & 10 & & $9.01 \pm 0.14$ & 7 & & \\
\hline & $8.53_{-0.13}^{+0.03}$ & 1 & $-0.09_{-0.10}^{+0.19}$ & 1 & NGC 2249 & $8.82 \pm 0.30$ & 4 & $\sim-0.47$ & 10 \\
\hline & $8.78_{-0.08}^{+0.04}$ & 1 & $-0.25_{-0.18}^{+0.10}$ & 1 & & & & & \\
\hline & $8.50 \pm 0.14$ & 7 & & & & $8.44 \pm 0.30$ & 8 & $-0.40 \pm 0.02$ & 8 \\
\hline \multirow[t]{4}{*}{ NGC 1868} & $8.74 \pm 0.30$ & 4 & $-0.50 \pm 0.20$ & 12 & & $8.54 \pm 0.10$ & 6 & & \\
\hline & $8.95 \pm 0.03$ & 9 & $-0.40 \pm 0.10$ & 9 & SL 506 & $9.26_{-0.11}^{+0.09}$ & 5 & $-0.66 \pm 0.20$ & 12 \\
\hline & $8.97 \pm 0.04$ & 8 & $-0.32 \pm 0.71$ & 8 & & $9.23 \pm 0.10$ & 9 & $-0.40 \pm 0.20$ & 9 \\
\hline & $8.87 \pm 0.10$ & 6 & & & SL 663 & $9.51_{-0.07}^{+0.06}$ & 13 & $-0.60 \pm 0.20$ & 12 \\
\hline \multirow[t]{6}{*}{ NGC 2121} & $9.51_{-0.07}^{+0.06}$ & 13 & $-0.61 \pm 0.20$ & 12 & & & & $-0.60 \pm 0.20$ & 13 \\
\hline & & & $-0.60 \pm 0.20$ & 13 & & $\sim 9.60$ & 14 & $\sim-1.0$ & 14 \\
\hline & $9.40_{-0.09}^{+0.08}$ & 11 & $-0.65 \pm 0.20$ & 11 & & & & & \\
\hline & $9.38 \pm 0.07$ & 11 & $-0.5 \pm 0.2$ & 11 & & & & & \\
\hline & $9.60 \pm 0.14$ & 7 & & & & & & & \\
\hline & $\sim 9.60$ & 14 & $\sim-1.0$ & 14 & & & & & \\
\hline \multirow[t]{8}{*}{ NGC 2155} & $9.51_{-0.07}^{+0.06}$ & 13 & $-0.55 \pm 0.20$ & 12 & & & & & \\
\hline & & & $-0.60 \pm 0.20$ & 13 & & & & & \\
\hline & $\sim 9.45$ & 2 & -0.98 to -0.80 & 2 & & & & & \\
\hline & $9.43 \pm 0.26$ & 8 & $-0.44 \pm 0.86$ & 8 & & & & & \\
\hline & $\sim 9.56$ & 11 & $\sim-0.80$ & 11 & & & & & \\
\hline & $9.46 \pm 0.05$ & 16 & $\sim-0.7$ & 16 & & & & & \\
\hline & $9.45 \pm 0.14$ & 7 & & & & & & & \\
\hline & $\sim 9.60$ & 14 & $\sim-1.0$ & 14 & & & & & \\
\hline
\end{tabular}

Reference list (technique): 1 - Beasley et al. (2002) (integrated spectra); 2 - Bertelli et al. (2003) (VLT/CMD); 3 - Dirsch et al. (2000) (CMD); 4 - Elson \& Fall (1988) (CMD); 5 - Geisler et al. (1997) (CMD); 6 - Girardi et al. (1995) (CMD); 7 - Girardi \& Bertelli (1998)(integrated colours); 8 - Leonardi \& Rose (2003) (integrated spectra) 9 - Kerber \& Santiago (2005) (HST/CMD); 10 - Mackey \& Gilmore (2003) (crude estimation based the $[\mathrm{Fe} / \mathrm{H}]$ from others clusters with similar ages); 11 - Piatti et al. (2003b) (CMD); 12 - Olszewski et al. (1991) (spectroscopy of red giants); 13 - Rich et al. (2001) (HST/CMD); 14 - Sarajedini (1998) (HST/CMD); 15 - Sarajedini et al. (2002) (CMD); 16 - Woo et al. (2003) (VLT/CMD).

LMC cluster NGC 1818. We expect that these reasonable assumptions for $\alpha$ and $f_{\text {bin }}$ combined with a regular model grid should prevent biases in the determination of the parameter space for each cluster.

\section{Statistical tools}

The physical parameters of each cluster were determined by statistical comparisons between synthetic CMDs from a grid of models and the observed one. This method combines a CMD modelling process that has two very important qualities: i) it potentially mimics the effects of photometric uncertainties and unresolved binaries, therefore realistically reproducing the observed CMD features; ii) it is based on objective criteria to determine which models best reproduce the data. This is a robust approach that avoids the subjectivity inherent to visual isochrone fits and that is able to reveal any model solutions that may go undetected in this simpler and more popular method.

There are several papers devoted to establishing such statistical tools, both in the context of CSPs (Gallart et al. 1999;
Hernandez et al. 1999; Dolphin 2002) and SSPs (Valls-Gabaud \& Lastennet 1999; Kerber et al. 2002; Kerber \& Santiago 2005). Since our data are not very deep, here we prefer to avoid a two-dimensional comparison method (which uses star counts throughout the CMD plane). Rather, we follow a more simplistic and appropriate approach that makes use of both the MS ridge line and the RC position. This approach is less sensitive to photometric uncertainties and incompleteness. The simultaneous comparison of these two CMD features ensures a reliable criterion to establish what the best models are, as demonstrated by control experiments.

For each CMD, an MS ridge line was determined using the $(B-V)$ median positions at each $V$ magnitude bin along the MS. Figure $5 \mathrm{ab}$ presents two synthetic CMDs and their MS fiducial lines computed in this way. They can be visually compared in panel c. To minimize contamination by spurious objects (certainly present in the observed CMDs) and stars belonging to other CMD branches, only stars inside the two lines as shown in this figure were considered as part of the MS. 


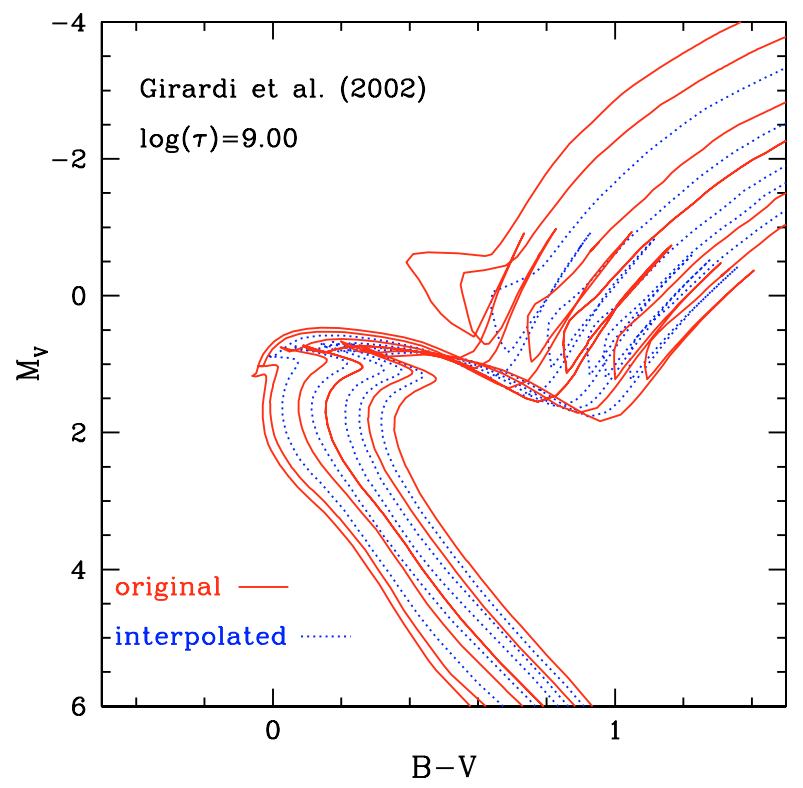

Fig. 4. Original isochrones (solid lines) from Girardi et al. (2002) and interpolated ones (dotted lines) using TRILEGAL code (Girardi et al. 2005).

The $\chi^{2}$ statistic was employed to compare the model (mod) and data (obs) colours, being computed for the $N_{\text {bin }}$ magnitude bins along the MS according to the expression

$\chi^{2}=\frac{1}{N_{\mathrm{bin}}-1} \sum_{i=1}^{N_{\mathrm{bin}}}\left[\frac{(B-V)_{\mathrm{obs}, i}-(B-V)_{\mathrm{mod}, i}}{\sigma_{\mathrm{B}-\mathrm{V}, \mathrm{MS}}}\right]^{2}$,

where $\sigma_{\mathrm{B}-\mathrm{V}, \mathrm{MS}}$ is the dispersion in the median colour position for the $i$ th $V$ magnitude bin in the model (typically $\sim 0.01 \mathrm{mag}$, as determined by control experiments).

The RC position was determined by using the median position in the CMD plane of the stars that likely belong to this phase and that fall inside an appropriately chosen CMD box. Therefore the $(V, B-V) \mathrm{RC}$ coordinates were determined by the median in the $V$ magnitude and colour distributions, respectively. This process is also depicted in Fig. 5.

To compare the $\mathrm{RC}$ positions, we define a distance on the CMD plane, given by

$\delta_{\mathrm{RC}}=\sqrt{\left[\frac{V_{\mathrm{obs}}-V_{\mathrm{mod}}}{\sigma_{V, \mathrm{RC}}}\right]^{2}+\left[\frac{(B-V)_{\mathrm{obs}}-(B-V)_{\mathrm{mod}}}{\sigma_{B-V, \mathrm{RC}}}\right]^{2}}$

where $\sigma_{V, \mathrm{RC}}$ and $\sigma_{B-V, \mathrm{RC}}$ are the dispersions in $V$ and $(B-V)$ coordinates for the model RC median position $(\sim 0.03$ and $\sim 0.01$, respectively).

We considered as the best models those that simultaneously satisfy the following criteria:

$\chi^{2} \leq \chi_{\min }^{2}+n \sigma_{\chi}$

$\delta_{\mathrm{RC}} \leq \delta_{\mathrm{RC}, \min }+n \sigma_{\delta}$,

where the index "min" refers to the model with the minimum value of each statistic, and $\sigma_{\chi}$ and $\sigma_{\delta}$ are respectively the expected dispersions in the distribution of $\chi^{2}$ and $\delta$ values. They were determined by comparing synthetic CMDs of the same model (both dispersions are usually $\sim 1.0$ to 2.0 ). The parameter " $n$ " is the necessary integer number of $\sigma$ in each statistic so that at least three models satisfy the criteria given above. As

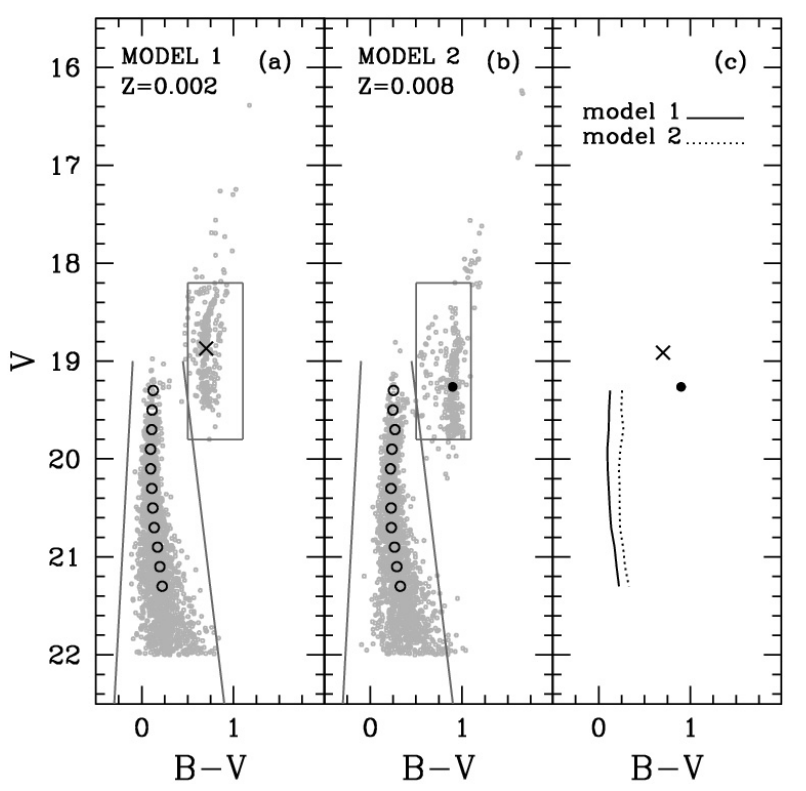

Fig. 5. Synthetic CMDs and their associated MS ridge lines and RC positions. As labelled in the panels, the models adopt different $Z$ values. Both CMDs were generated using the following parameters: $\log (\tau / \mathrm{yr})=$ 9.00, $(m-M)_{0}=18.50, E(B-V)=0.05$.

shown in Sect. 6, $n$ is typically 2 or 3 . However, in some cases, solutions were found only with higher values of $n$, indicating less reliable solutions. The two statistics have the same weight in the determination of the best models, meaning that the MS and the RC are equally important in the final solution for each cluster.

To test our statistical approach and to quantify the formal uncertainties associated with it we ran some control experiments where synthetic CMDs were used as "observed CMDs" with known input parameters. The results from these experiments are detailed in the appendix. As expected, for each experiment the best models recovered by our statistical tools have similar parameters when compared to the model used to create the "observation".

The value of each physical parameter derived for a cluster was assumed as the mean of its best models, the associated uncertainty being the maximum value among the following ones: i) the dispersion calculated for the best models; ii) the formal uncertainty, as determined by a control experiment (see Appendix); iii) the half bin size in the model grid.

\section{Results}

The resulting physical parameters of each cluster are presented in Table 2, including the conversion of $\log (\tau)$ and $Z$ to $\tau$ and $[\mathrm{Fe} / \mathrm{H}]$ values to help with future comparisons and use of these parameters. In the last column the parameter $n$ is presented to reflect the reliability of the result.

In Figs. 6-20 we compare the observed and synthetic CMDs. The sequence of presentation is the same as in Fig. 1 to underline the evolutionary sequence for these clusters. In all figures, panel a presents the data and panel b shows a synthetic CMD generated from one of the best models according to the criteria discussed in the previous section. The corresponding fiducial lines and RC positions are compared in panel c. For 11 clusters we have $n \leq 3$ and, in general, the models reproduce well both the MS fiducial line, the RC position and the spread in magnitude 
Table 2. Physical parameters derived for all clusters.

\begin{tabular}{lccccccc}
\hline \hline Cluster & $\log (\tau / \mathrm{yr})$ & $\tau / \mathrm{Gyr}$ & $Z$ & {$[\mathrm{Fe} / \mathrm{H}]$} & $(m-M)_{0}$ & $E(B-V)$ & $n(\sigma)$ \\
\hline NGC 1651 & $9.30 \pm 0.03$ & $2.00 \pm 0.15$ & $0.004 \pm 0.001$ & $-0.70 \pm 0.10$ & $18.53 \pm 0.03$ & $0.11 \pm 0.01$ & 2 \\
NGC 1718 & $9.31 \pm 0.03$ & $2.05 \pm 0.15$ & $0.008_{-0.001}^{+0.002}$ & $-0.40 \pm 0.10$ & $18.73 \pm 0.07$ & $0.10 \pm 0.03$ & 3 \\
NGC 1777 & $9.06 \pm 0.04$ & $1.15 \pm 0.15$ & $0.005 \pm 0.001$ & $-0.60 \pm 0.10$ & $18.56 \pm 0.07$ & $0.10 \pm 0.03$ & 4 \\
NGC 1831 & $8.85 \pm 0.05$ & $0.70 \pm 0.10$ & $0.016 \pm 0.003$ & $-0.10 \pm 0.10$ & $18.23 \pm 0.09$ & $0.01 \pm 0.02$ & 7 \\
NGC 1856 & $8.47 \pm 0.04$ & $0.30 \pm 0.25$ & $0.008_{-0.001}^{+0.002}$ & $-0.40 \pm 0.10$ & $18.37 \pm 0.10$ & $0.21 \pm 0.02$ & 2 \\
NGC 1868 & $9.05 \pm 0.03$ & $1.10 \pm 0.10$ & $0.004 \pm 0.001$ & $-0.70 \pm 0.10$ & $18.33 \pm 0.06$ & $0.04 \pm 0.01$ & 3 \\
NGC 2121 & $9.46 \pm 0.07$ & $2.90 \pm 0.50$ & $0.008_{-0.001}^{+0.002}$ & $-0.40 \pm 0.10$ & $18.24 \pm 0.04$ & $0.07 \pm 0.02$ & 5 \\
NGC 2155 & $9.48 \pm 0.03$ & $3.00 \pm 0.25$ & $0.004 \pm 0.001$ & $-0.70 \pm 0.10$ & $18.32 \pm 0.04$ & $0.02 \pm 0.01$ & 2 \\
NGC 2162 & $9.10 \pm 0.03$ & $1.25 \pm 0.10$ & $0.008 \pm 0.002$ & $-0.40 \pm 0.10$ & $18.35 \pm 0.08$ & $0.03 \pm 0.02$ & 3 \\
NGC 2173 & $9.21 \pm 0.04$ & $1.60 \pm 0.20$ & $0.005 \pm 0.001$ & $-0.60 \pm 0.10$ & $18.58 \pm 0.12$ & $0.07 \pm 0.02$ & 2 \\
NGC 2209 & $9.08 \pm 0.03$ & $1.20 \pm 0.10$ & $0.006 \pm 0.001$ & $-0.50 \pm 0.10$ & $18.43 \pm 0.09$ & $0.15 \pm 0.03$ & 4 \\
NGC 2213 & $9.23 \pm 0.04$ & $1.70 \pm 0.20$ & $0.004 \pm 0.001$ & $-0.70 \pm 0.10$ & $18.56 \pm 0.08$ & $0.06 \pm 0.03$ & 2 \\
NGC 2249 & $9.00 \pm 0.03$ & $1.00 \pm 0.10$ & $0.007 \pm 0.001$ & $-0.45 \pm 0.10$ & $18.27 \pm 0.06$ & $0.01 \pm 0.02$ & 2 \\
SL 506 & $9.35 \pm 0.03$ & $2.25 \pm 0.15$ & $0.007 \pm 0.001$ & $-0.45 \pm 0.10$ & $18.48 \pm 0.06$ & $0.08 \pm 0.03$ & 2 \\
SL 663 & $9.50 \pm 0.05$ & $3.15 \pm 0.40$ & $0.004 \pm 0.001$ & $-0.70 \pm 0.10$ & $18.32 \pm 0.07$ & $0.07 \pm 0.02$ & 3 \\
\hline
\end{tabular}

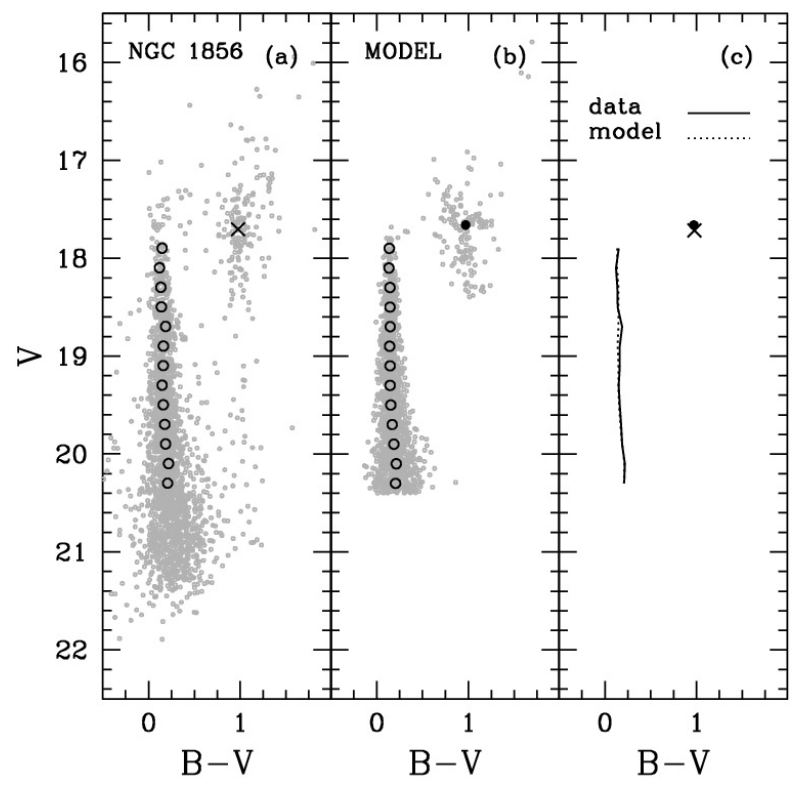

Fig. 6. Data (panel a) vs. model (panel b) comparison for NGC 1856. Panel $\mathbf{c}$ confronts the fiducial lines and $\mathrm{RC}$ positions traced by the points shown in panel a) and b). The synthetic CMD was generated using the following parameters: $\log (\tau / \mathrm{yr})=8.45, Z=0.008,(m-M)_{0}=18.45$, $E(B-V)=0.21$.

and colour. However, in a few cases there are some discrepancies. They will be commented on below together with the cases where $n>3$. In the next subsections we will also compare our age and metallicity results with the ones found in the literature summarized in Table 1.

In terms of distance modulus and reddening there is a lack direct determinations for a significant number of clusters in our sample. The only recent exception to this rule is the work by McLaughlin \& van der Marel (2005), who compared SSP model predictions with integrated colours. As uncertainties based on integrated cluster light tend to be larger, we prefer to use the results of McLaughlin \& van der Marel (2005) to assess the extent to which their method reproduces the results of resolved photometry rather than to assess the reliability of our method itself. Typical LMC values adopted for $E(B-V)$ and reddening are $\sim 0.07$ (Burstein \& Heiles 1982; Schlegel et al. 1998) and $\sim 18.50$ (see Clementini et al. 2003, for a review about the distance to the

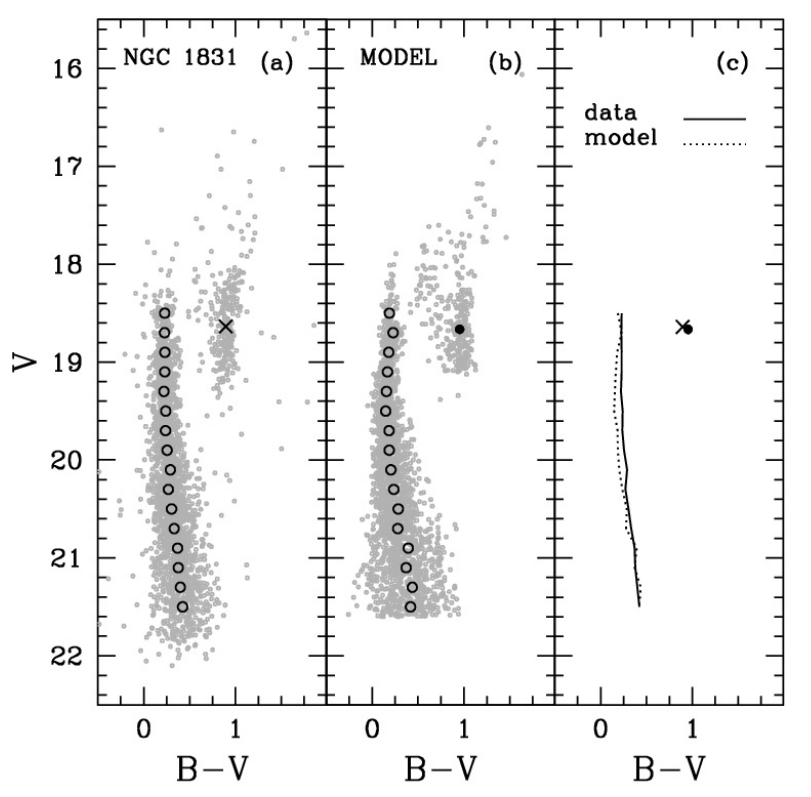

Fig. 7. The same as in Fig. 6 but for NGC 1831. The synthetic CMD was generated using the following parameters: $\log (\tau / \mathrm{yr})=8.85, Z=0.016$, $(m-M)_{0}=18.20, E(B-V)=0.00$.

LMC). Thus, in the next section we will comment only on results that deviate strongly from these fiducial values.

\subsection{Cluster-by-cluster}

\section{NGC 1856}

This is the youngest cluster and the nearest to the LMC optical centre in our sample, which explains the high reddening value obtained, in accordance with the ones recovered by McLaughlin \& van der Marel (2005) (typically $E(B-V) \simeq$ $0.20 \pm 0.05)$. Notice that the synthetic CMD reproduces well both the MS fiducial line and RC features. However, the model did not reproduce the stars located beyond the upper limit of the MS, which are likely binaries and/or blue stragglers.

Our age result is consistent with the upper limit of Elson \& Fall (1988) and with the one obtained by Girardi et al. (1995). It is also consistent with the lowest age solution from Beasley et al. (2002). However these authors recovered an almost solar metallicity, higher than the value inferred here. As expected from the age-metallicity degeneracy, their highest age solution has 


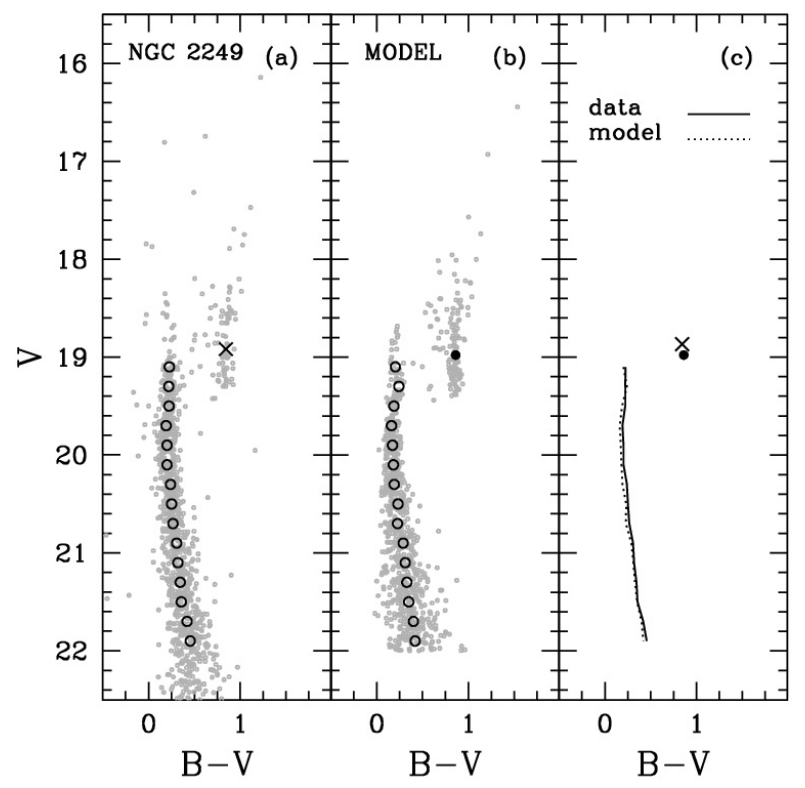

Fig. 8. The same as in Fig. 6 but for NGC 2249. The synthetic CMD was generated using the following parameters: $\log (\tau / \mathrm{yr})=9.00, Z=0.008$, $(m-M)_{0}=18.25, E(B-V)=0.00$.

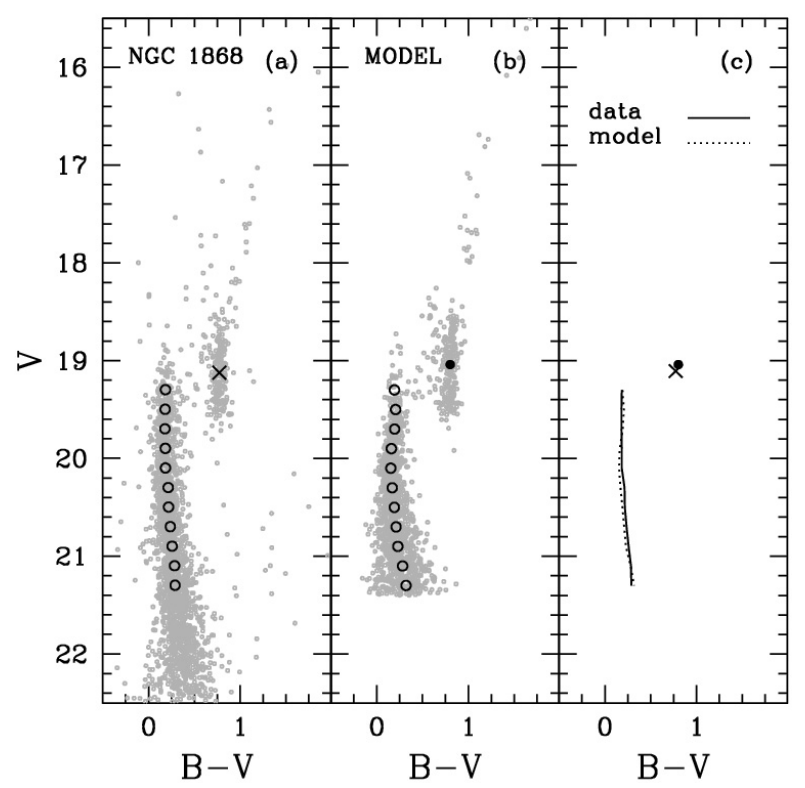

Fig. 9. The same as in Fig. 6 but for NGC 1868. The synthetic CMD was generated using the following parameters: $\log (\tau / \mathrm{yr})=9.05, Z=0.004$, $(m-M)_{0}=18.35, E(B-V)=0.04$.

a lower value for metallicity, compatible with our value within the uncertainties.

\section{NGC 1831}

Only solutions with $n=7$, i.e., the best 3 models spread out in a region $7 \times \sigma$ away from the minimum $\chi^{2}$, were found for the second youngest cluster in our sample. The difficulty here was to find models with the appropriate color difference between the MS fiducial line and RC position. The best models still have bluer MS fiducial lines and redder RC positions than the data. On the other hand, these solutions visually mimic the stars at the bright end of the MS and the RC spread.

Our results confirm a high metallicity value, also found by OSSH (using only one star) and Kerber \& Santiago (2005), although this latter work recovered a slightly younger age than

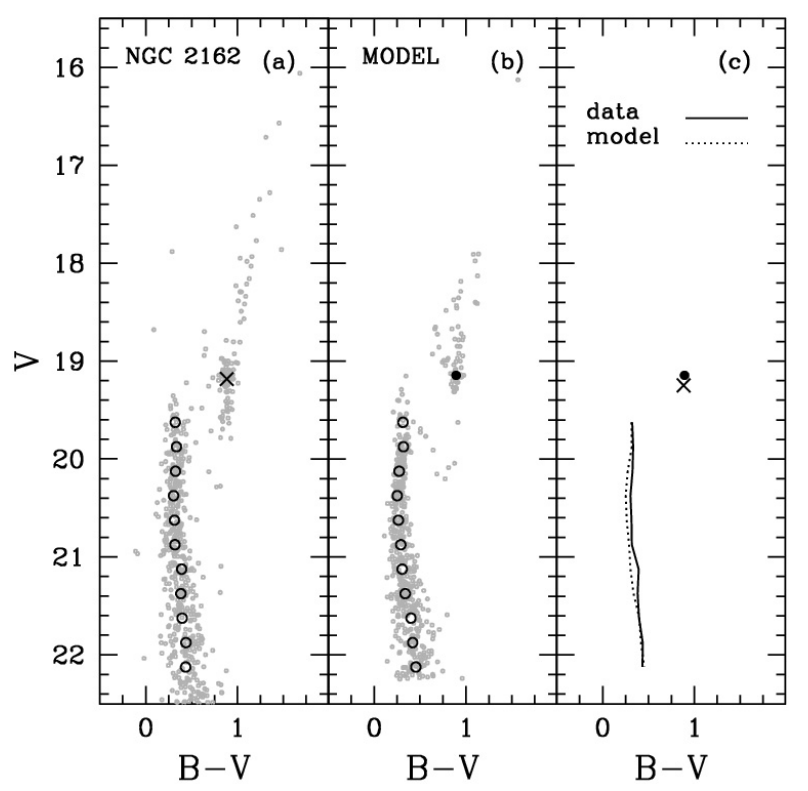

Fig. 10. The same as in Fig. 6 but for NGC 2162. The synthetic CMD was generated using the following parameters: $\log (\tau / \mathrm{yr})=9.10, Z=$ $0.008,(m-M)_{0}=18.35, E(B-V)=0.03$.

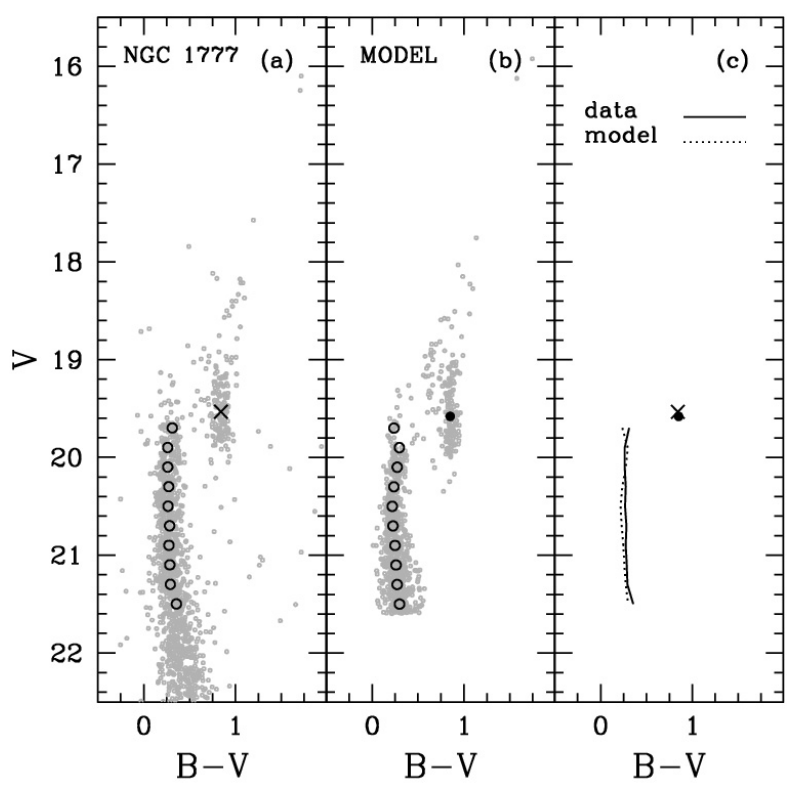

Fig. 11. The same as in Fig. 6 but for NGC 1777. The synthetic CMD was generated using the following parameters: $\log (\tau / \mathrm{yr})=9.05, Z=$ $0.004,(m-M)_{0}=18.55, E(B-V)=0.11$.

found here. The upper age limits obtained by Elson \& Fall (1988) and Leonardi \& Rose (2003) are consistent with our result, although the metallicity recovered by the latter is significantly lower.

As determined by Kerber \& Santiago (2005), we found a low reddening value, but they derived a larger distance modulus $(18.70 \pm 0.03)$ than obtained here. This and other discrepancies between the results in this work and those obtained by Kerber \& Santiago (2005) are likely due to two main reasons: i) here we analyse not only the MS, but also the RC, a very important phase which, when combined with the MS, better constrains the best models (see Appendix for control experiments); ii) the systematic biases found in the photometry in both studies were not 


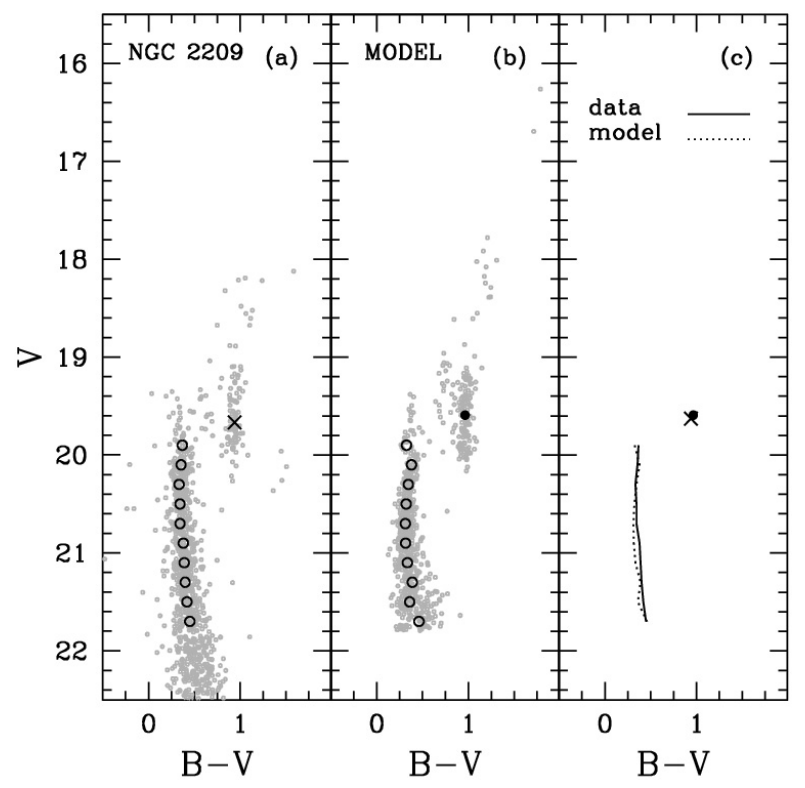

Fig. 12. The same as in Fig. 6 but for NGC 2209. The synthetic CMD was generated using the following parameters: $\log (\tau / \mathrm{yr})=9.05, Z=$ $0.006,(m-M)_{0}=18.40, E(B-V)=0.16$.

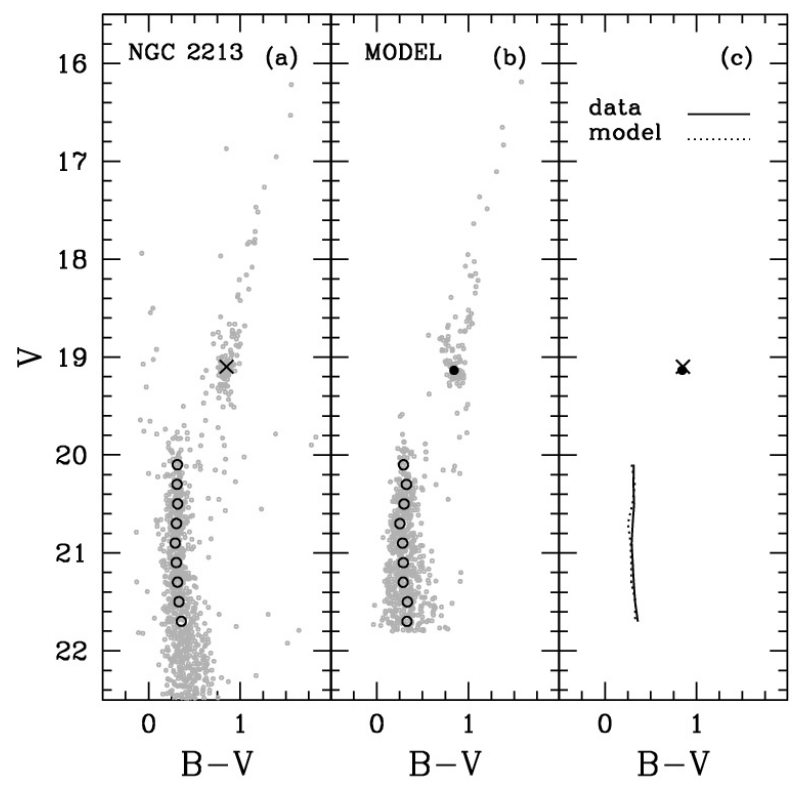

Fig. 13. The same as in Fig. 6 but for NGC 2213. The synthetic CMD was generated using the following parameters: $\log (\tau / \mathrm{yr})=9.20, Z=$ $0.004,(m-M)_{0}=18.55, E(B-V)=0.06$.

corrected by the same method, likely leading to some photometric discrepancies.

\section{NGC 2249}

The agreement between data and model is close for this cluster. Our recovered age is only in agreement with Elson \& Fall (1988), the other previous values being significantly younger. Our metallicity result is in accordance with those by Leonardi \& Rose (2003) and Mackey \& Gilmore (2003). We reach low reddening and distance modulus values. As most of the extinction is internal to the LMC, clusters seen in the foreground should have lower reddening.

\section{NGC 1868}

Again the best models reproduced well the MS fiducial line and the RC characteristics.

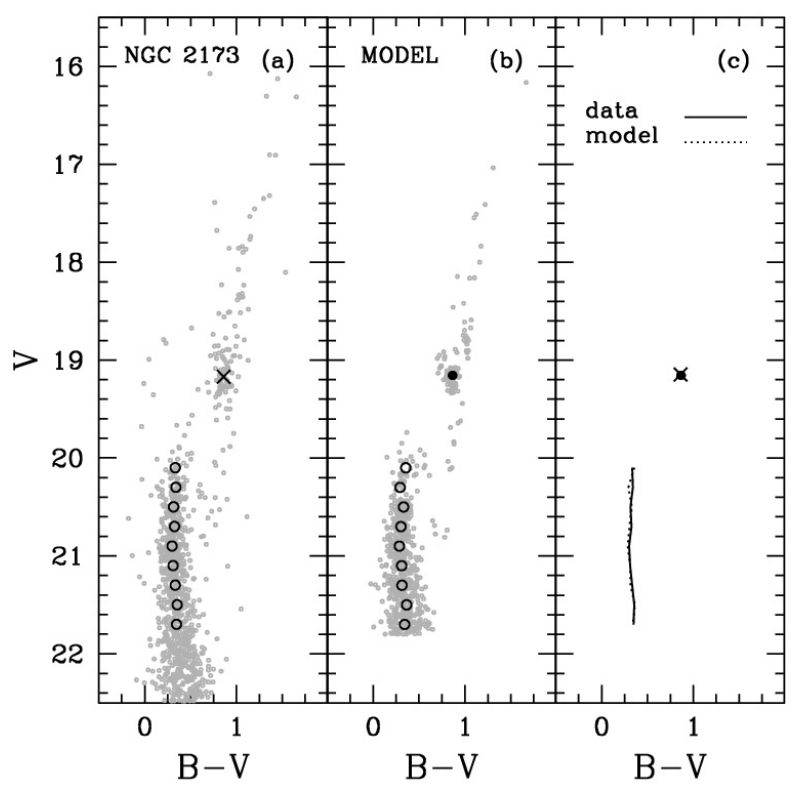

Fig. 14. The same as in Fig. 6 but for NGC 2173. The synthetic CMD was generated using the following parameters: $\log (\tau / \mathrm{yr})=9.20, Z=$ $0.004,(m-M)_{0}=18.60, E(B-V)=0.07$.

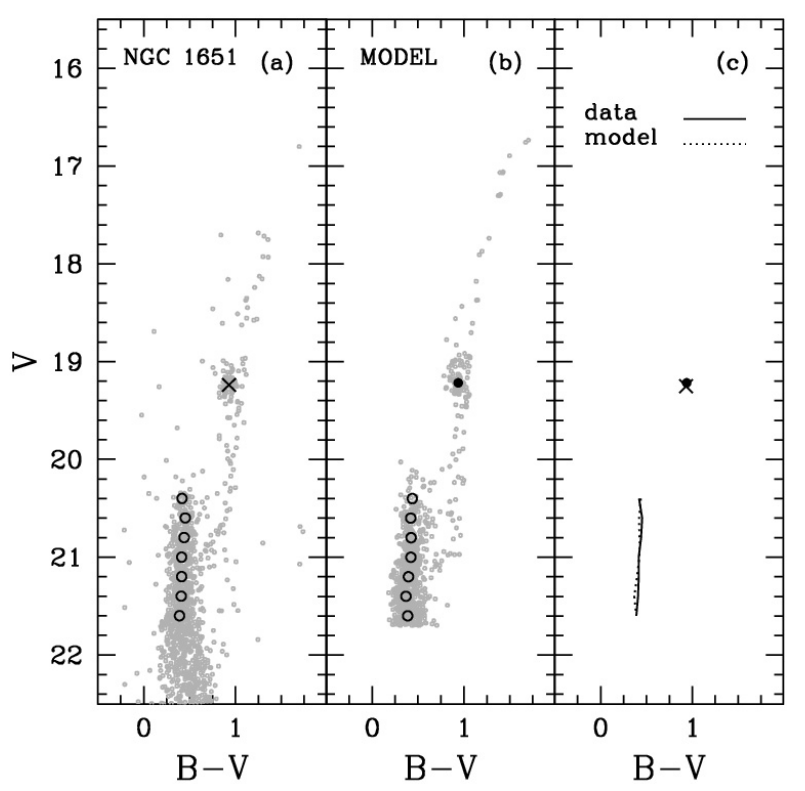

Fig. 15. The same as in Fig. 6 but for NGC 1651. The synthetic CMD was generated using the following parameters: $\log (\tau / \mathrm{yr})=9.30, Z=$ $0.004,(m-M)_{0}=18.50, E(B-V)=0.11$.

The ages found in the literature are systematically younger than ours, although the upper limits of Elson \& Fall (1988), Leonardi \& Rose (2003) and Kerber \& Santiago (2005) are consistent with our determination. The metallicity we obtain is consistent with those from OSSH and Leonardi \& Rose (2003) (although this latter is highly uncertain), but it is lower than the one recovered by Kerber \& Santiago (2005).

As in NGC 1831, we obtained a low reddening value, as was found by Kerber \& Santiago (2005), but again our best models have a significantly smaller distance modulus.

\section{NGC 2162}

The best solutions correctly fit the RC position, but have MS fiducial lines slightly bluer than the data. These solutions are limited by $n=3$. The recovered age matches the results 


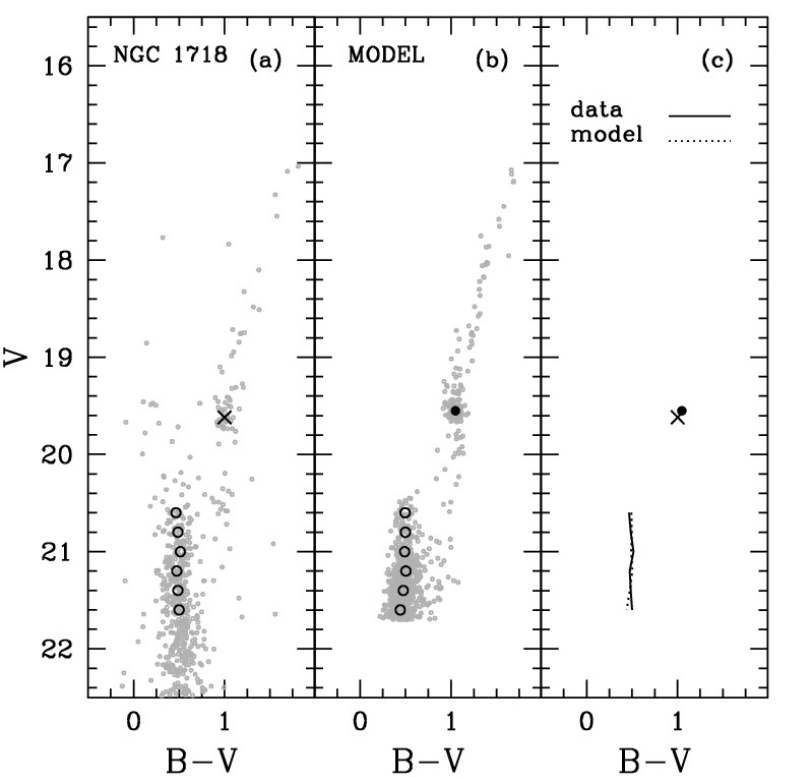

Fig. 16. The same as in Fig. 6 but for NGC 1718. The synthetic CMD was generated using the following parameters: $\log (\tau / \mathrm{yr})=9.30, Z=$ $0.008,(m-M)_{0}=18.70, E(B-V)=0.10$.

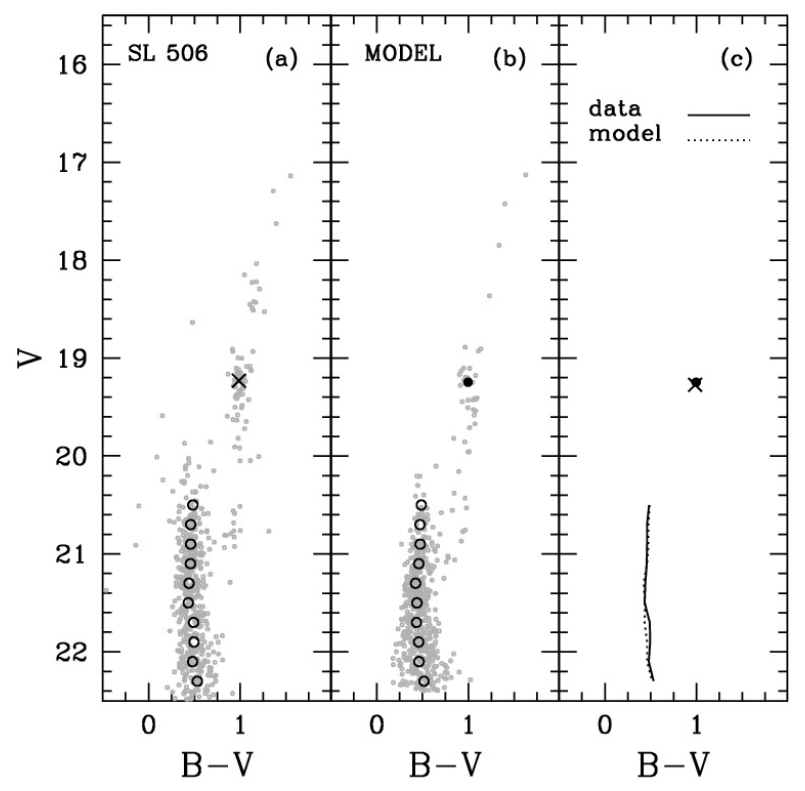

Fig. 17. The same as in Fig. 6 but for SL 506. The synthetic CMD was generated using the following parameters: $\log (\tau / \mathrm{yr})=9.35, Z=0.008$, $(m-M)_{0}=18.50, E(B-V)=0.05$.

by Geisler et al. (1997) and the upper limit of Girardi et al. (1995). Our inferred metallicity agrees with OSSH within the uncertainties. The solution found by Leonardi \& Rose (2003) is significantly older and more metal-poor than most other results. Low reddening and distance modulus values were found for NGC 2162, again consistent with a foreground location relative to most LMC stars.

\section{NGC 1777}

Although the best solutions require $n=4$, they visually reproduce all the observed features in the CMD. The age result is in good agreement with previous determinations. Our recovered metallicity is consistent with the lower limit determined by OSSH and by Leonardi \& Rose (2003), if one admits a more

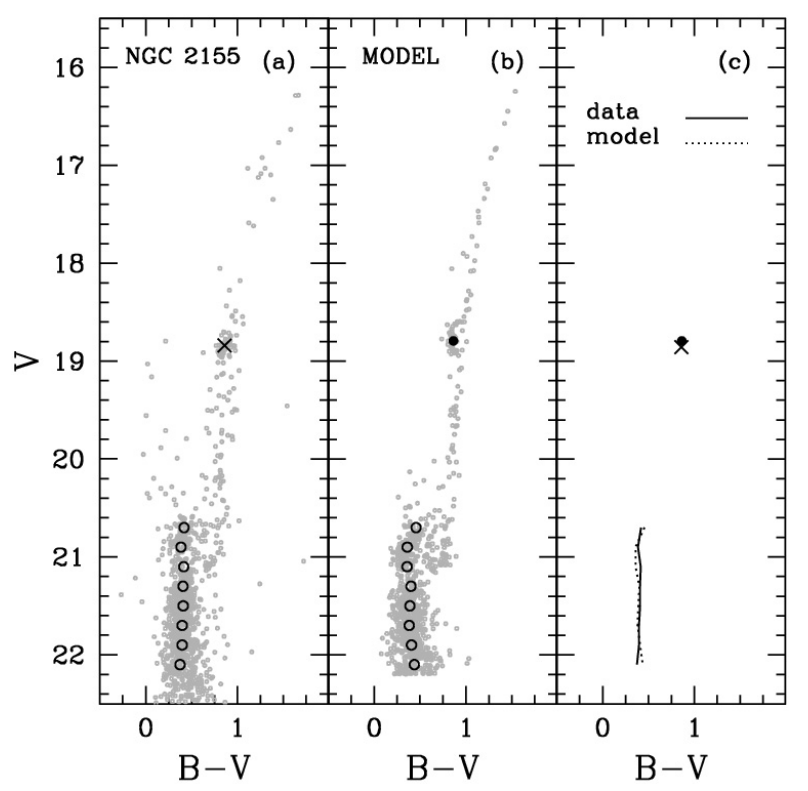

Fig. 18. The same as in Fig. 6 but for NGC 2155. The synthetic CMD was generated using the following parameters: $\log (\tau / \mathrm{yr})=9.50, Z=$ $0.004,(m-M)_{0}=18.30, E(B-V)=0.02$.

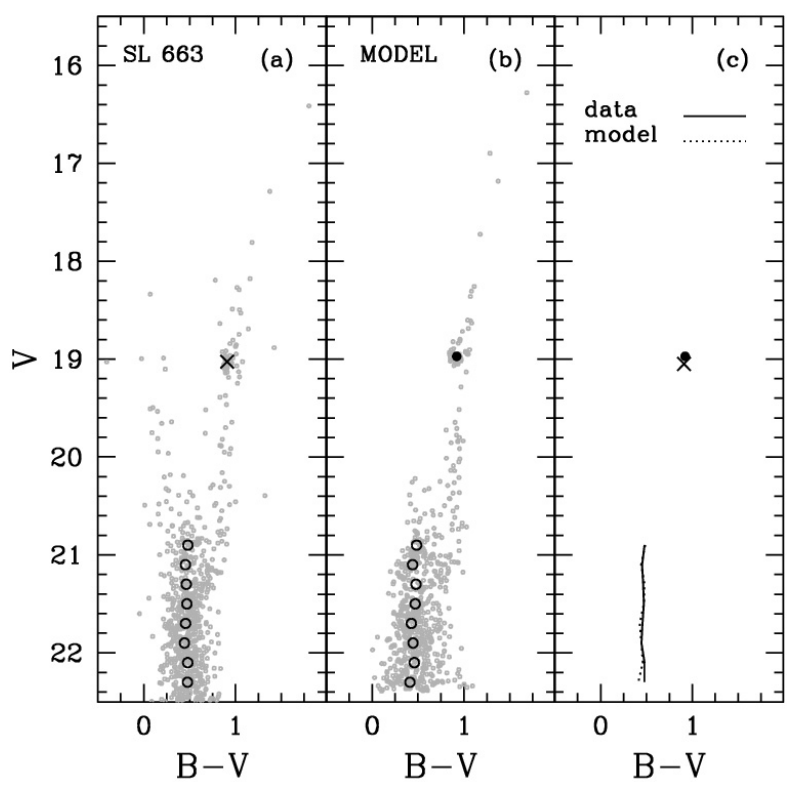

Fig. 19. The same as in Fig. 6 but for SL 663. The synthetic CMD was generated using the following parameters: $\log (\tau / \mathrm{yr})=9.45, Z=0.004$, $(m-M)_{0}=18.35, E(B-V)=0.08$.

typical (and likely more realistic) uncertainty of $\sim 0.10$ for the value from the latter.

\section{NGC 2209}

The best models correctly reproduce the RC features and nicely mimic the unresolved binaries in the MS termination. The $n=4 \sigma$ level of these solutions is related to the difficulty in reproducing the MS fiducial line, since the models are slightly but systematically bluer than the data.

The ages found in the literature agree with our result. Unfortunately we did not find a unique published metallicity determination for this cluster, but the crude estimate done by Mackey \& Gilmore (2003) closely agrees with our result, meaning that this cluster has a typical metallicity for an IAC. 


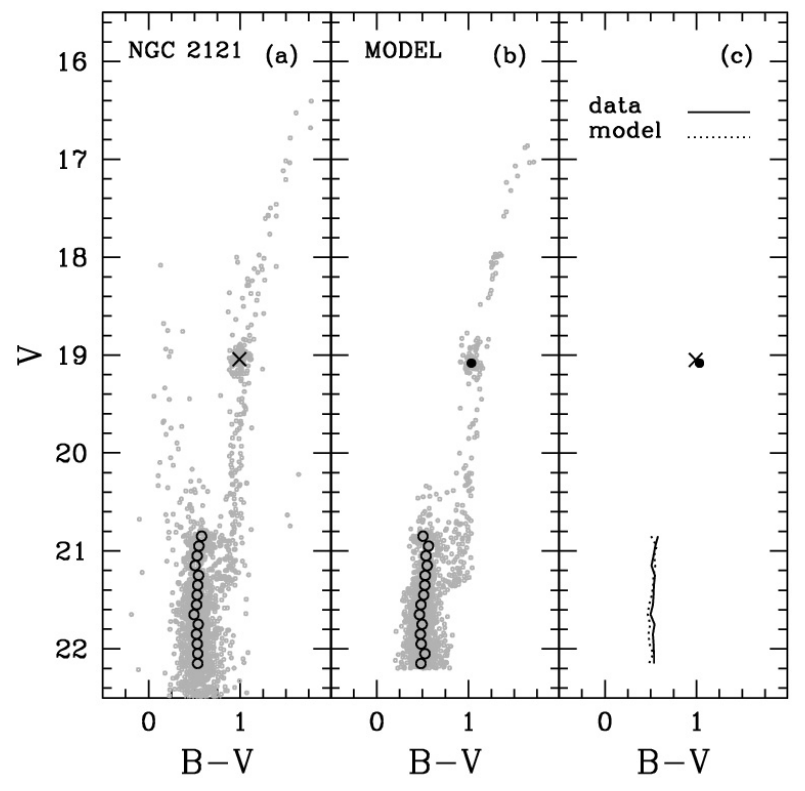

Fig. 20. The same as in Fig. 6 but for NGC 2121. The synthetic CMD was generated using the following parameters: $\log (\tau / \mathrm{yr})=9.45, Z=$ $0.008,(m-M)_{0}=18.25, E(B-V)=0.07$.

A high reddening value was determined for this cluster, although it is located at a distance modulus slightly lower than the typical LMC value $\left((m-M)_{0}=18.50\right)$. McLaughlin \& van der Marel (2005) derived $E(B-V)$ values that are typically half of what we infer here, but with large uncertainties $(\sim 0.10)$.

\section{NGC 2213}

The agreement between model and data CMDs is very close for this cluster, including the RGB and the onset of the SGB. Our age estimate also agrees well with that of Geisler et al. (1997), but it is lower than that from Leonardi \& Rose (2003) and higher than the one from Girardi \& Bertelli (1998). If one accepts a more typical uncertainty of $\sim 0.10$ in $\log (\tau)$ than that quoted by Leonardi \& Rose (2003), their value becomes compatible with our one. The metallicity result from OSSH is much higher than our value, but this spectroscopic result was based on only one star.

\section{NGC 2173}

This is another example of a quality fit between data and model, which also adequately reproduces the observed SGB and RGB. The only weak point seems to be the RC spread, lower in the model than in the data.

A large number of results are found in the literature. Our age determination is consistent with Geisler et al. (1997) and with the recent works done by Bertelli et al. (2003) (although they argued that this cluster had a prolonged star formation) and Woo et al. (2003), being higher than the one derived by Girardi et al. (1995) and significantly lower than the one obtained by Leonardi \& Rose (2003). However, if the result from Leonardi \& Rose (2003) is correct, then this cluster would be the second one located in the age gap. Also, these authors find a very low metallicity compared to a typical IAC. On the other hand, the higher metallicity value from OSSH is not confirmed by the two independent solutions found by Woo et al. (2003) and Bertelli et al. (2003), which are based on VLT/CMDs and which use $Y^{2}$ and Padova isochrones, respectively.

The distance modulus and reddening for this cluster were also derived by Bertelli et al. (2003) and Woo et al. (2003). While the latter found results consistent with ours, the former obtained a distance modulus $\sim 0.10$ lower than the one found by us; however, their acceptable solutions, based on different criteria, presented some internal discrepancies in the reddening value (and in the metallicity), their intermediate reddening solution (with $Z=0.003$ ) being in accordance with ours.

\section{NGC 1651}

Both the MS fiducial line and the RC features are well reproduced by the models. However, the SGB in the synthetic CMD seems to be more scattered and the RGB more elongated towards the bright end than in the observed CMD.

The age results found in the literature are in accordance with each other and with this work. Taking the uncertainties into account, our resulting metallicity agrees with the previous metalpoor determinations from Dirsch et al. (2000) and Leonardi \& Rose (2003). The result from OSSH is metal-richer than ours, but it was based on a single star, whereas the metallicity derived by Sarajedini et al. (2002) is, surprisingly, almost solar.

Recently the distance modulus was determined by Sarajedini et al. (2002) and Grocholski et al. (2005), analysing the RC stars with near infrared data. They found $(m-M)_{0}=18.55 \pm$ 0.12 and $(m-M)_{0}=18.50 \pm 0.06$, respectively, in accordance with our result. Notice also that their adopted reddening value, based on Burstein \& Heiles (1982) and Schlegel et al. (1998), was $E(B-V)=0.12 \pm 0.02$, again in agreement with our determination.

\section{NGC 1718}

Although the MS fiducial line has been well fitted by the best models, the model RC is slightly brighter than the data. Notice also the presence of stars near but above the MS termination, not reproduced by the models, that are probable unresolved binaries. Another group of stars well above the MSTO may be field stars, possibly belonging to horizontal branch. The best models still visually reproduce very well both the SGB and RGB, supporting our solutions.

Our determinations for age agree with the results from Elson \& Fall (1988) and with the lowest age solution found by Beasley et al. (2002). However, only the upper limit predicted for metallicity by these latter authors is compatible with our value. As expected, this discrepancy in metallicity becomes greater if we compare our result with that from the highest age solution found by Beasley et al. (2002). On the other hand, our derived metallicity is typical of an IAC in the LMC, as attested by the metallicity estimate by Mackey \& Gilmore (2003).

We infer for this cluster the highest distance modulus in the sample and a moderately high value of reddening. Although less accurate, the reddening results from McLaughlin $\&$ van der Marel (2005) $(\sim 0.10 \pm 0.10)$ point in the same direction.

\section{SL 506 (Hodge 14)}

The observed CMD of this cluster is well reproduced by the best models in all its features. Although the two previous results from the literature have slightly younger ages, they are still consistent. Our determination also agrees very well with metallicity values of Kerber \& Santiago (2005) and are consistent with OSSH, who found a lower value.

In terms of distance modulus, Kerber \& Santiago (2005) reached almost the same result obtained here. Their reddening value $(E(B-V)=0.02 \pm 0.02)$ is marginally consistent with ours, taking into account the uncertainties in the two determinations.

\section{NGC 2155}

Again the best models well reproduce both the MS fiducial line and the RC, but the RGB in the synthetic CMDs seems to be narrower than in the observed CMD. Above the MSTO it is also 


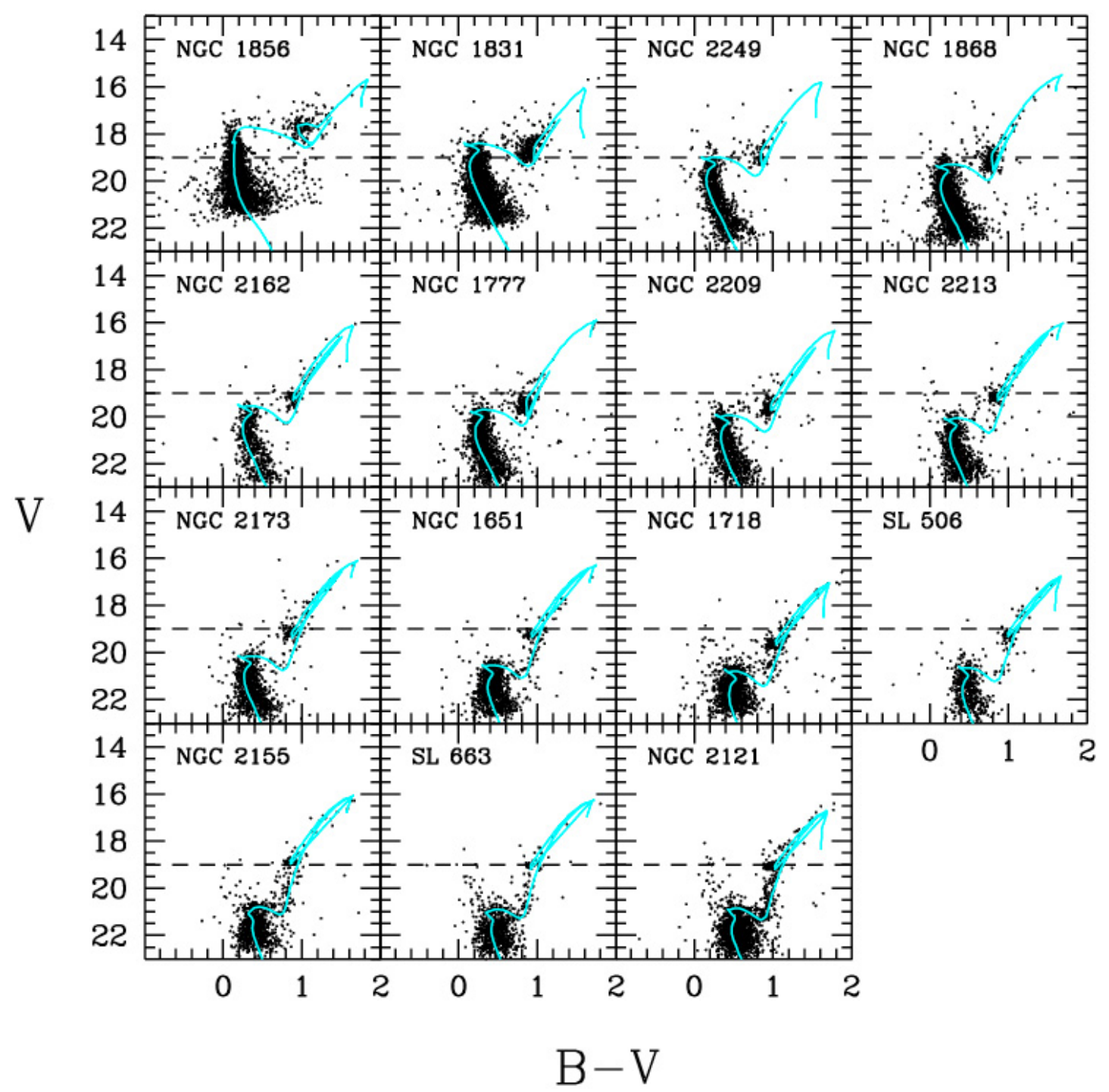

Fig. 21. All observed CMDs, as shown in Fig. 1, but with the isochrones corresponding to the best solutions superimposed.

possible to identify some likely unresolved binaries (mimicked by the artificial CMDs) and field stars ( $\gtrsim 0.60$ mag brighter than the MS termination). This cluster has a large number of age and metallicity determinations, and our results are in agreement with them.

We obtained a clear self-consistent result for the distance modulus and reddening, both being low values, again indicating a foreground location. This result is in agreement with Bertelli et al. (2003), who obtained their best solutions with $(m-M)_{0}=$ 18.36 and $E(B-V) \sim 0.015-0.028$. On the other hand, Woo et al. (2003) derived a distance modulus typical of the LMC (18.50), 0.20 higher than our value, but they also recovered a low reddening value $(E(B-V) \sim 0.04)$. This low reddening value seems to be confirmed by the Burstein \& Heiles (1982) maps, which indicate $E(B-V)=0.03$ for this region in the sky.

\section{SL 663}

The observed MS fiducial line and RC features are well fitted by the best models. As for NGC 2155, there are some likely field stars and unresolved binaries beyond the MSTO, these latter also being reproduced by the artificial CMDs. The less populated RGB of this cluster is also reproduced by the models. Our age and metallicity results agree well both with Rich et al. (2001) and OSSH.

\section{NGC 2121}

Although they visually reproduce the observed CMD, the best models have $n \leq 5$. As for other previous clusters, the likely field star contamination and the presence unresolved binaries can be seen. Since this cluster is more populous, its RGB is correspondingly more populated, something that the model CMDs manage to recover.

The age we obtain is in good agreement with those found in the literature. Our inferred metallicity is slightly higher than the typical $[\mathrm{Fe} / \mathrm{H}] \sim-0.6$ value, but it is consistent with it when the uncertainties are considered.

According to our results, this cluster has the lowest $(m-M)_{0}$ in the sample, but a typical $E(B-V)$ value for the LMC.

We present the observed CMDs of all clusters, as in Fig. 1, but now with the best model isochrones superposed. The isochrones from the best solutions are those presented in panels b of Figs. 6-20. This is shown in Fig. 21, which reveals, in general, good isochrone fits to the data, both for MS and RC position. As expected due to its high $n$ value, the only conspicuous exception is NGC 1831. In some cases (e.g. NGC 2249) the isochrone is systematically shifted bluewards relative to the bulk of the MS stars. This shift is expected to compensate for the effect of unresolved binaries, since these stars tend to spread the 


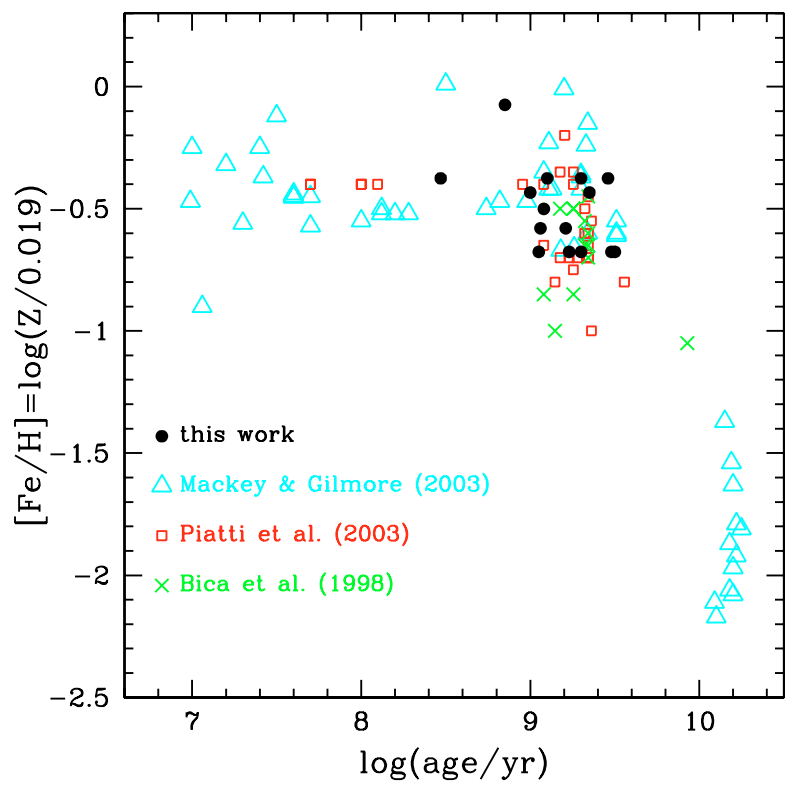

Fig. 22. Age-metallicity relation from this work (black points) in comparison to the one by Mackey \& Gilmore (2003) (open triangles) and the one obtained by Bica et al. (1998) (crosses).

MS in the redward direction. The effect is also less prominent for a steeper MS. The unresolved binaries are also responsible for blurring the bright MS termination, since a pair of equal mass stars will reach 0.75 mag brighter than the expected, single-star, MS termination magnitude determined by the isochrone line.

\subsection{The whole cluster sample}

We compare the properties we infer for the whole cluster sample with other previous homogeneous determinations found in the literature.

Figure 22 presents the age-metallicity relation (AMR) for a series of works devoted to LMC clusters (Bica et al. 1998; Mackey \& Gilmore 2003; Piatti et al. 2003b) spanning a wide age and metallicities ranges. Our results for the 15 IACs are also plotted in this figure. This reveals the LMC chemical enrichment: the oldest clusters have the lowest metallicities values (reaching $[\mathrm{Fe} / \mathrm{H}] \sim-2.3)$, whereas the clusters younger than $\log (\tau / \mathrm{yr}) \lesssim 9.5(\tau \lesssim 3 \mathrm{Gyr})$ are significantly more metal-rich, belonging to an approximated "plateau" of $[\mathrm{Fe} / \mathrm{H}] \sim-0.5$, but with a considerable scatter. The only known cluster in the so-called "age gap" range $(9.5 \lesssim \log (\tau) \lesssim 10.0$ or $3.0 \lesssim \tau \lesssim 10 \mathrm{Gyr})$ is ESO121-SC03 (marked by a cross, as determined by Bica et al. 1998). This gap can also be considered as a "metallicity gap" for clusters within the range $-1.3 \lesssim[\mathrm{Fe} / \mathrm{H}] \lesssim-1.0$.

To better understand our contribution to the AMR, we selected the region covered by our cluster sample, comparing our results with the ones summarized by Mackey \& Gilmore (2003) and by Leonardi \& Rose (2003). These comparisons are presented in Figs. 23 and 24, respectively. Mackey \& Gilmore (2003) is a good compilation of results based mainly on ages determined by CMD analysis and metallicities from the OSSH spectroscopic study of red giants (with some exceptions, as shown in Table 1). Leonardi \& Rose (2003) is a study based on integrated spectra. We are comparing here only results for clusters in common, which means 15 objects in the case of Mackey \& Gilmore (2003) and 9 objects in the case of Leonardi \& Rose (2003).

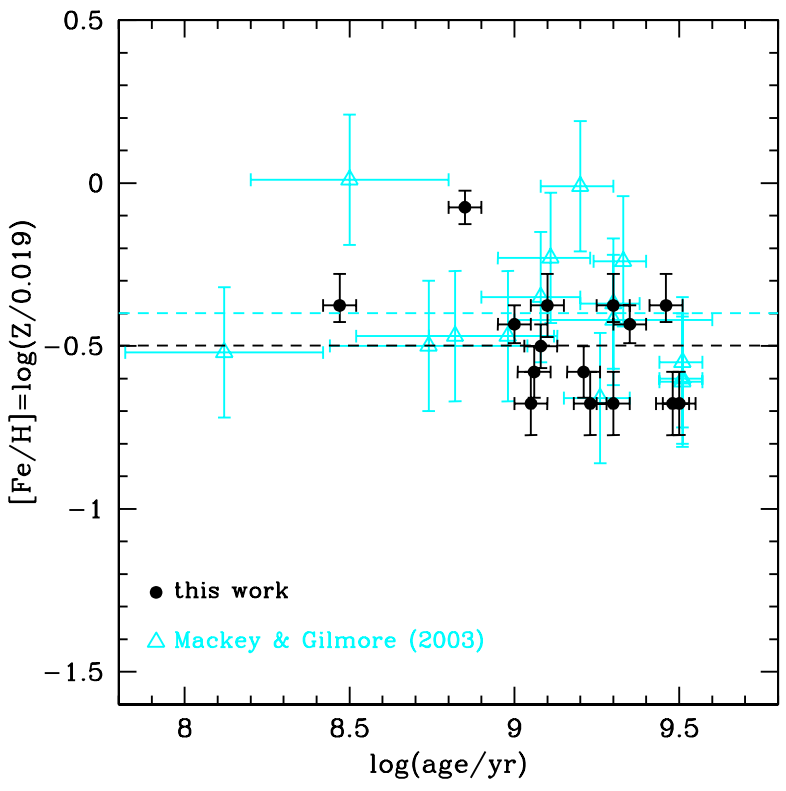

Fig. 23. Age-metallicity relation from this work (black) in comparison to the one by Mackey \& Gilmore (2003) (gray) for the same clusters. The dotted (dashed) line represents our (their) mean values.

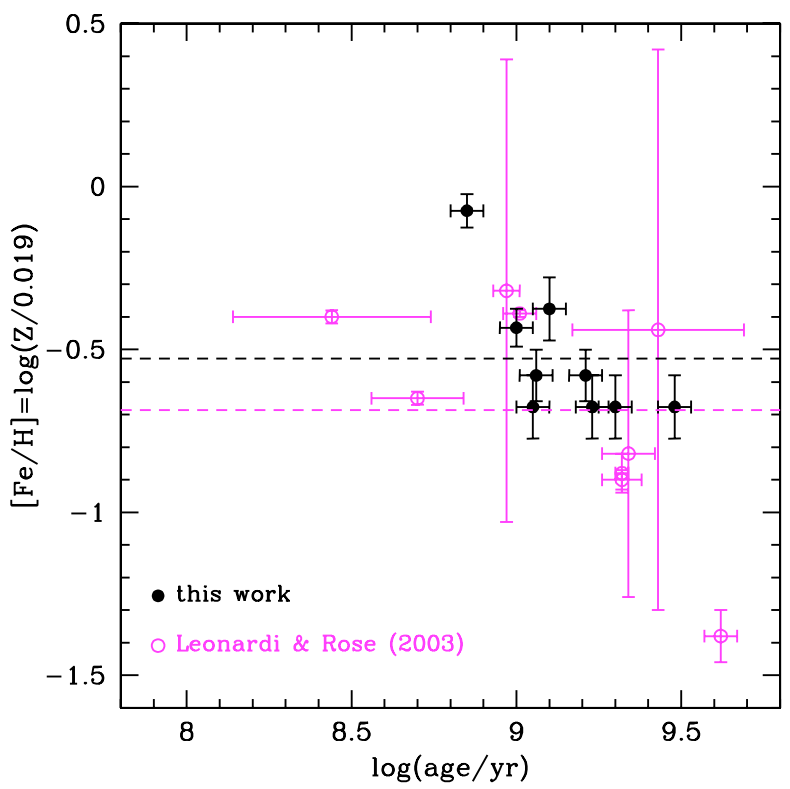

Fig. 24. Age-metallicity relation from this work (black) in comparison to that from Leonardi \& Rose (2003) (gray) for the same clusters. The dotted (dashed) line represents our (their) mean values.

In general, our results have smaller uncertainties, especially when ages are concerned. Our results also have a lower spread in metallicities $(\sigma=0.17)$ when compared with those considered by Mackey \& Gilmore (2003) (from OSSH) $(\sigma=0.24)$, and Leonardi \& Rose (2003) $(\sigma=0.34)$. Our mean metallicity in both comparisons is $[\mathrm{Fe} / \mathrm{H}] \sim-0.50$, lower than that from Mackey \& Gilmore $(2003)([\mathrm{Fe} / \mathrm{H}] \sim-0.40)$ and higher than the mean in Leonardi \& Rose $(2003)([\mathrm{Fe} / \mathrm{H}] \sim-0.70)$.

In the following two figures we compare our age results with the ones presented by Mackey \& Gilmore (2003) (Fig. 25) and by Leonardi \& Rose (2003), Girardi et al. (1995) and Girardi \& Bertelli (1998) (Fig. 26). As discussed, the ages given by Mackey \& Gilmore (2003) come from CMD analysis, mainly using ground-based data (Elson \& Fall 1988; Geisler et al. 1997) 


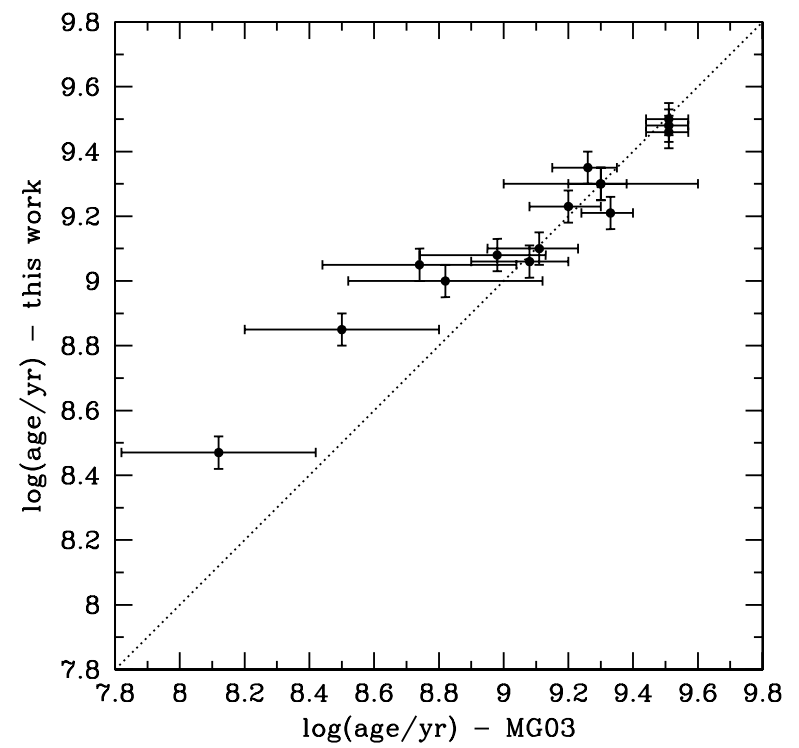

Fig. 25. Our age results in comparison to the ones by Mackey \& Gilmore (2003) for the same clusters. The identity relation is represented by the dotted line.

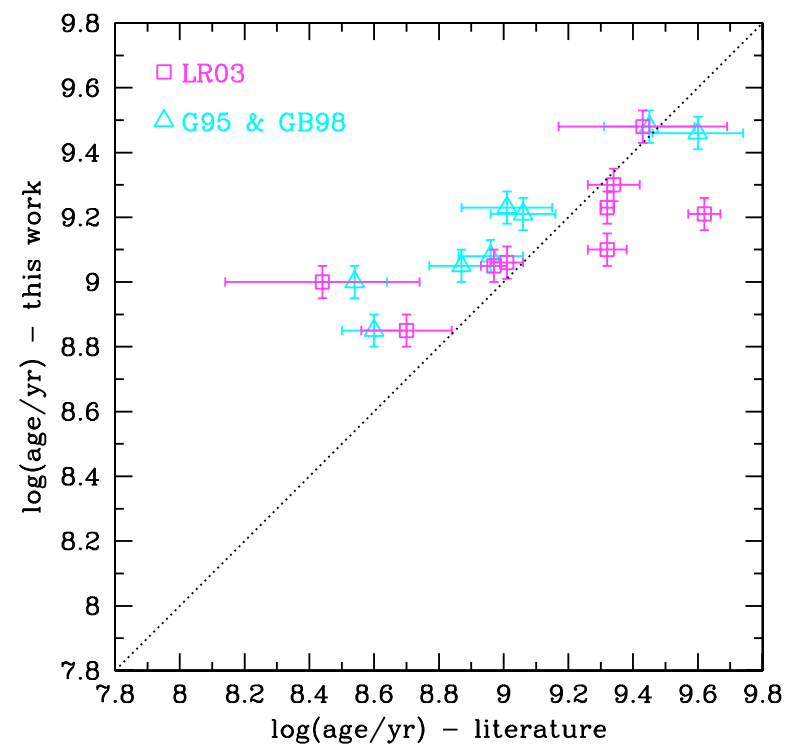

Fig. 26. Our age results in comparison to the ones from Leonardi \& Rose (2003), Girardi et al. (1995) and Girardi \& Bertelli (1998). The identity relation is represented by the dotted line.

but also using WFPC2/HST data (Rich et al. 2001) for the three oldest clusters. This comparison reveals a good agreement for clusters older than $\log (\tau / \mathrm{yr}) \sim 9.0$, especially for the ones with more accurate photometry from WFPC2/HST. On the other hand, for clusters younger than this limit our results systematically predict higher ages, but are still consistent with the upper limit of the results compiled by Mackey \& Gilmore (2003). This systematic trend is observed when we compare our determinations with the ones from Girardi et al. (1995) and Girardi \& Bertelli (1998), whereas the Leonardi \& Rose (2003) results also present significant discrepancies for old clusters. The trend towards higher age values for younger clusters was recently reported by Beasley et al. (2002), where their results for the only cluster in common with us (NGC 1856) seems to confirm this inference.

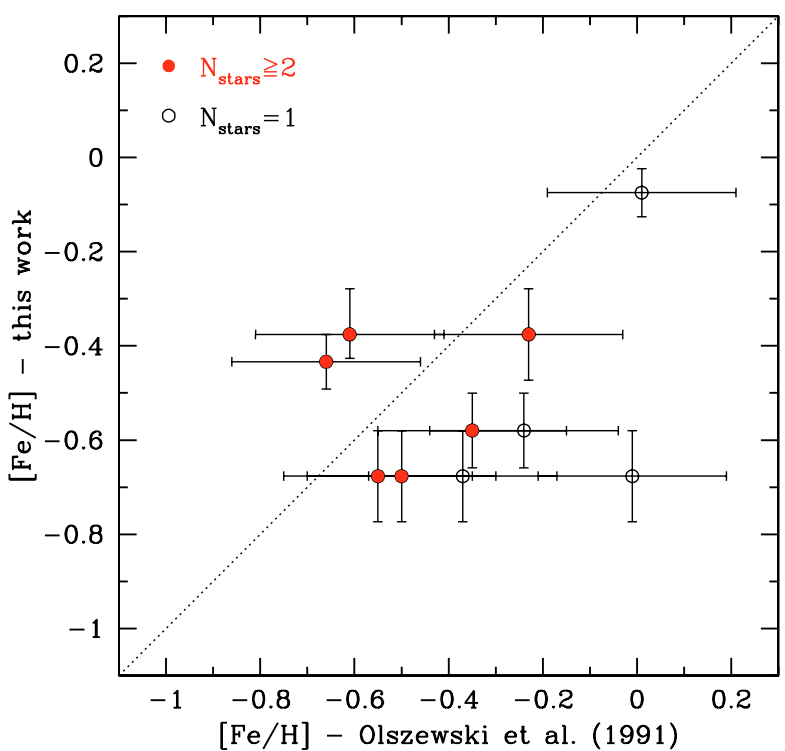

Fig. 27. Our $[\mathrm{Fe} / \mathrm{H}]$ values in comparison to the ones from OSSH. The identity relation is represented by the dotted line.

Concerning metallicity, Fig. 27 contrasts our results with the ones from OSSH (also selected by Mackey \& Gilmore 2003). A preliminary comparison indicates some discrepancies close to $[\mathrm{Fe} / \mathrm{H}] \sim-0.70$ (in our results), in the sense that OSSH determined higher values than us. However, these most discrepant points (NGC 1651, NGC 2173 and NGC 2213) come from determinations where only one star per cluster was used, therefore being subject to high uncertainties. Geisler recently showed at a FONDAP/ESO Conference (Globular Cluster - Guide to Galaxies) (Geisler 2006; Grocholski et al. 2006) new results for these clusters in agreement with our $[\mathrm{Fe} / \mathrm{H}]$ values. We also notice that the recalibrated OSSH values for the correcting transformation proposed by Cole et al. (2005) tend to slightly reduce the differences between metallicities (the mean difference between us and OSSH, 〈this work - OSSH $\rangle_{[\mathrm{Fe} / \mathrm{H}]}$, drops from -0.16 to $-0.10)$. Considering only the OSSH determinations based on more than one star per cluster, the mean metallicity becomes very similar in both samples $(\sim 0.50$, with an insignificant mean difference of $\left.\langle\text { this work - OSSH }\rangle_{[\mathrm{Fe} / \mathrm{H}]} \sim-0.02\right)$ and insensitive to the correction proposed by Cole et al. (2005).

Comparisons of our metallicity results with the ones obtained from CMD analysis and by Leonardi \& Rose (2003) are shown in Fig. 28. While the results based on CMDs reveal a satisfactory agreement, the Leonardi \& Rose (2003) ones present some discrepant points, in the sense that they determined significantly lower metallicities. However, the quoted uncertainties by these latter authors may be an underestimate, specially for a study based on integrated spectra.

We compare our reddening results with the ones obtained by McLaughlin \& van der Marel (2005) for the 14 clusters we have in common. These comparisons, shown in Fig. 29, reveal larger uncertainties in the McLaughlin \& van der Marel (2005) estimates, jeopardizing a more systematic comparison between them. However, our most discrepant reddening value (that of NGC 1856, which is significantly higher than the others) is in accordance with McLaughlin \& van der Marel (2005).

Since a relation between reddening and distance modulus is expected, we plot our results for these two parameters in Fig. 30. Except for some outlying points and less reliable estimates (open circles) a clear and consistent trend is revealed: more distant 


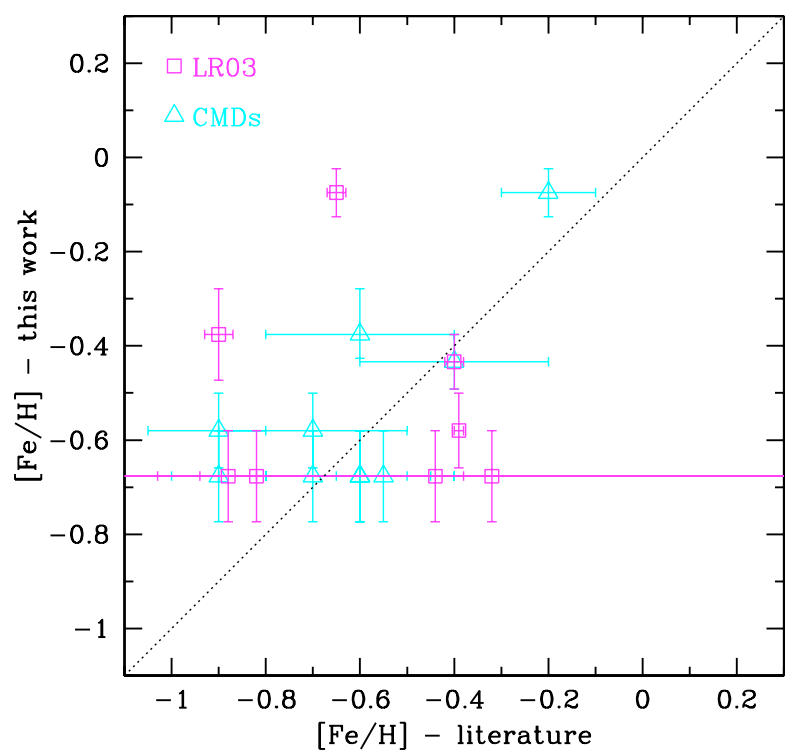

Fig. 28. Our $[\mathrm{Fe} / \mathrm{H}]$ values in comparison to the ones from CMDs and from Leonardi \& Rose (2003). The identity relation is represented by the dotted line.

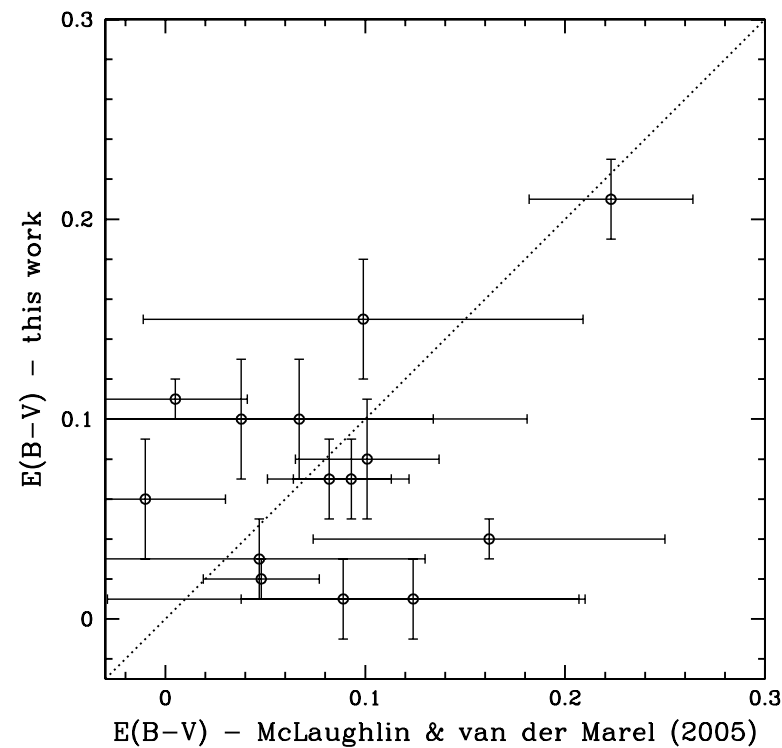

Fig. 29. Our reddening results in comparison to the ones from McLaughlin \& van der Marel (2005). The identity relation is represented by the dotted line.

clusters tend to be more reddened and vice-versa, as represented by the linear fit shown in this figure. Using this derived relation, a typical $E(B-V)$ value for the LMC is found ( $\sim 0.07)$ for the canonical LMC distance $\left((m-M)_{0}=18.50\right)$. This reddening value has an amplitude of $\sim 0.05$, possibly reflecting the variations in the LMC optical depth.

The on-sky distribution of the clusters (Figs. 31-32) reveals that the lowest reddening, closest and highest velocity (as determined by OSSH) ones are preferentially located in the NE region. On the other hand, the SW region seems to host clusters with the opposite characteristics.

This result for the spatial distribution of clusters agrees well with the geometry known for the LMC disk (Westerlund 1997) and recently revisited by Nikolaev et al. (2004) using Cepheids, who determined a position angle $(\theta)$ of $151.0^{\circ} \pm 2.4^{\circ}$ for the

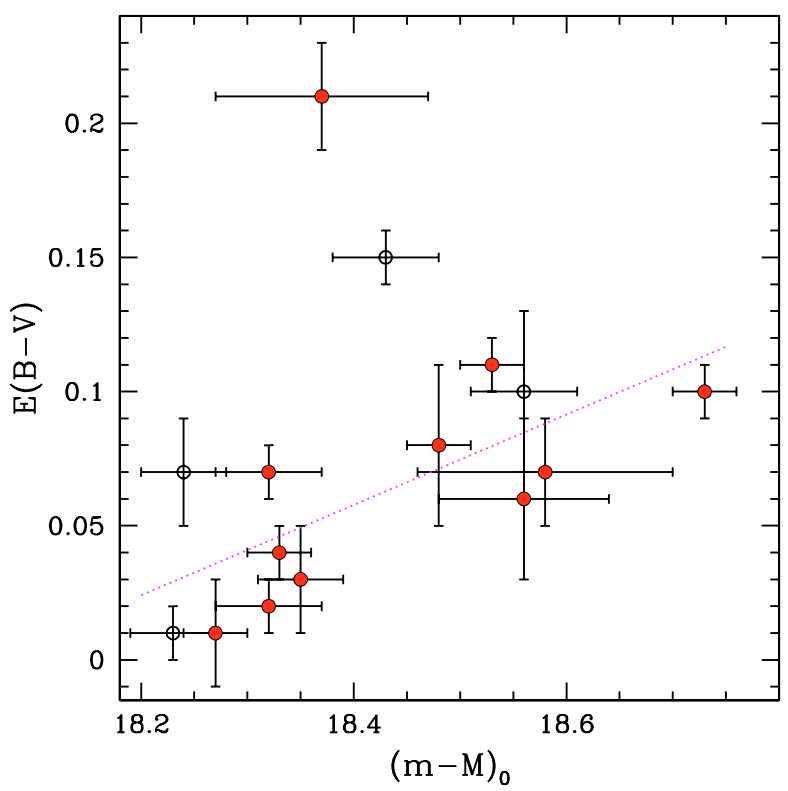

Fig. 30. Relation between our inferred reddening and distance modulus. Solid (open) circles are the best models confined to $n \leq 3(n>3)$, and therefore represent the most (least) reliable ones. The dotted line is the linear fit for the most reliable solutions excluding the highest reddening value.

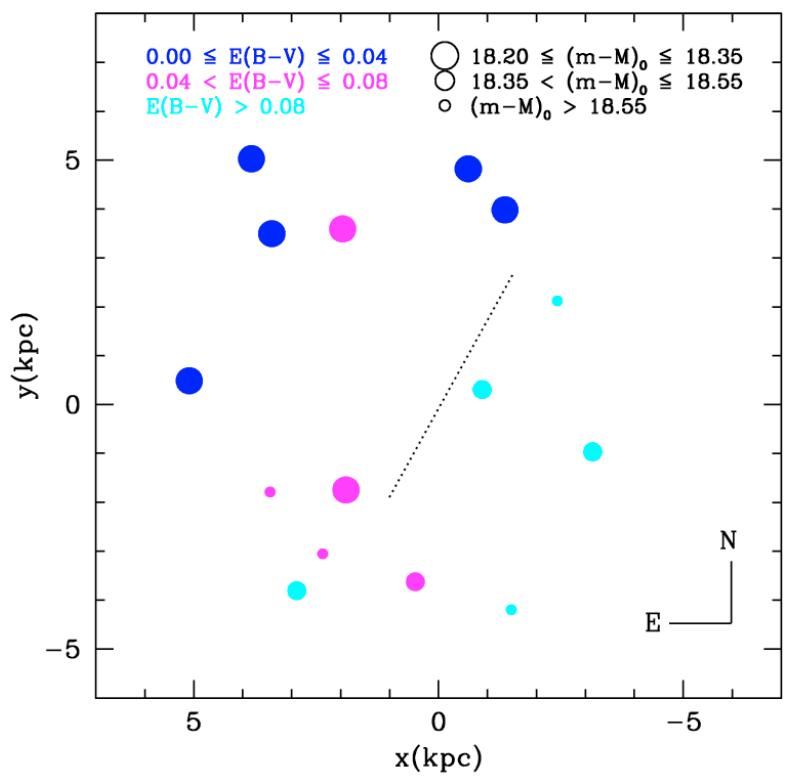

Fig. 31. Distribution of LMC clusters on the sky in physical units (as in Fig. 2, using the relation $1^{\circ}=0.873 \mathrm{kpc}$ corresponding to the $\left.(m-M)_{0}=18.50\right)$, with symbols coded according with their reddening (gray scale) and distance modulus (size). The dotted line indicates the line of nodes for the disk, as determined by Nikolaev et al. (2004). The distances are relative to the optical centre of the LMC bar (Bica et al. 1996).

line of nodes and $30.7^{\circ} \pm 1.1^{\circ}$ for the disk inclination $(i)$, with the northeast quadrant being the closest. Figure 33 illustrates this accordance showing the cluster positions on a plane for an imaginary observer with the line of sight aligned with the line of nodes for the disk. In other words, the $z$ axis for this plane is at the canonical distance to the LMC centre $\left((m-M)_{0}=18.50\right)$, while the $x^{\prime}$ axis is the perpendicular distance to the line of nodes. Although the cluster distribution is scattered, the clusters 


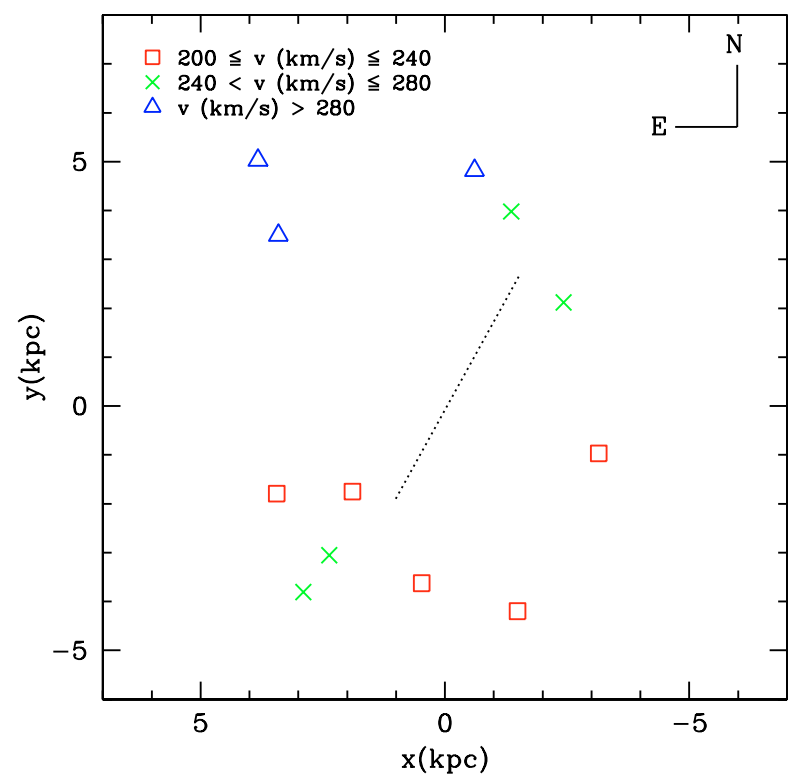

Fig. 32. Same as in Fig. 31, but with symbols coded according with their velocity, as determined by OSSH.

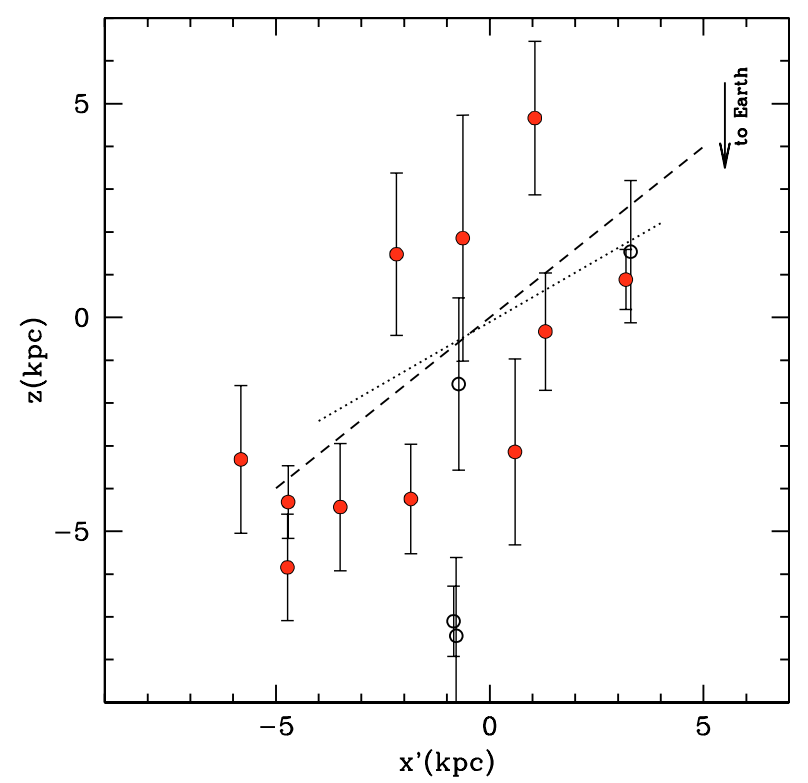

Fig. 33. Distribution of LMC clusters as seen by an imaginary observer whose $z$-axis is along the line of nodes. The dotted line indicates the projected LMC disk plane as determined by Nikolaev et al. (2004). The linear solution for the clusters with the most reliable results is shown by the dashed line. The direction to Earth is shown in the up right corner.

with the most reliable determinations have an inclination of $\sim 39^{\circ}$ $\left( \pm 7^{\circ}\right)$ and thus roughly follow the disk, with the closest clusters at the NE quadrant (negative $x^{\prime}$ values). The mean distance modulus calculated for the whole cluster sample is 18.42 (with a dispersion of 0.16 ), being slightly lower than the typical assumed LMC distance, likely reflecting a larger number of systems located in the NE quadrant than in the SW one. If we consider only the most reliable solutions, the mean and dispersion for the distance modulus remain almost the same, being 18.44 and 0.14 , respectively.

The radial velocities determined by OSSH relative to the mean radial velocity determined by Cole et al. (2005) $\left(256 \mathrm{~km} \mathrm{~s}^{-1}\right)$ are represented in this plane in Fig. 34. This

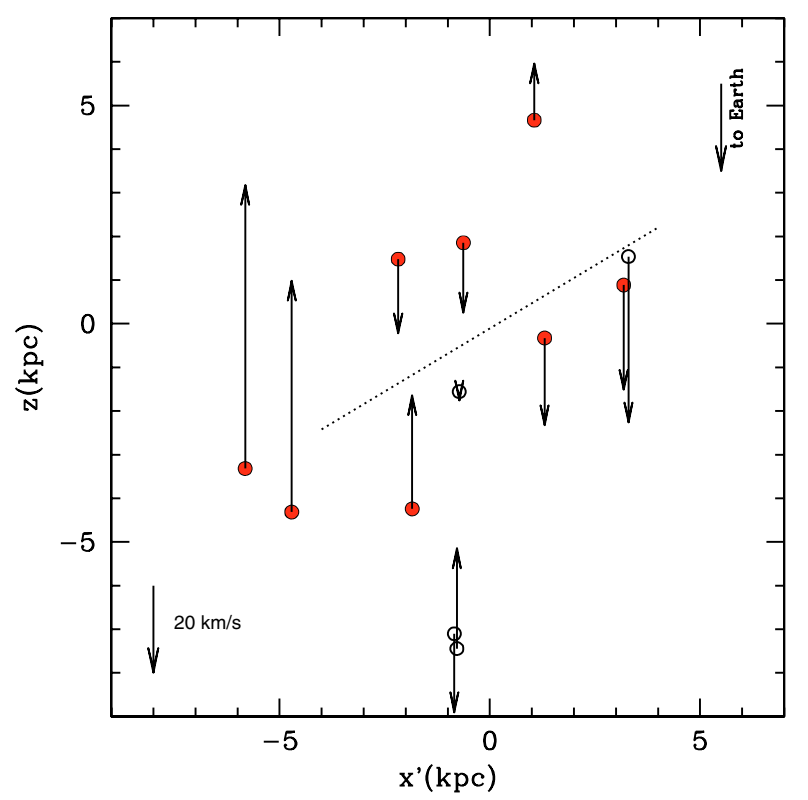

Fig. 34. Same as Fig. 33, but showing the radial velocity of each cluster, as determined by OSSH, relative to the mean radial velocity of stars belonging to the LMC bar as determined by Cole et al. (2005). A velocity scale is shown in the bottom left corner.

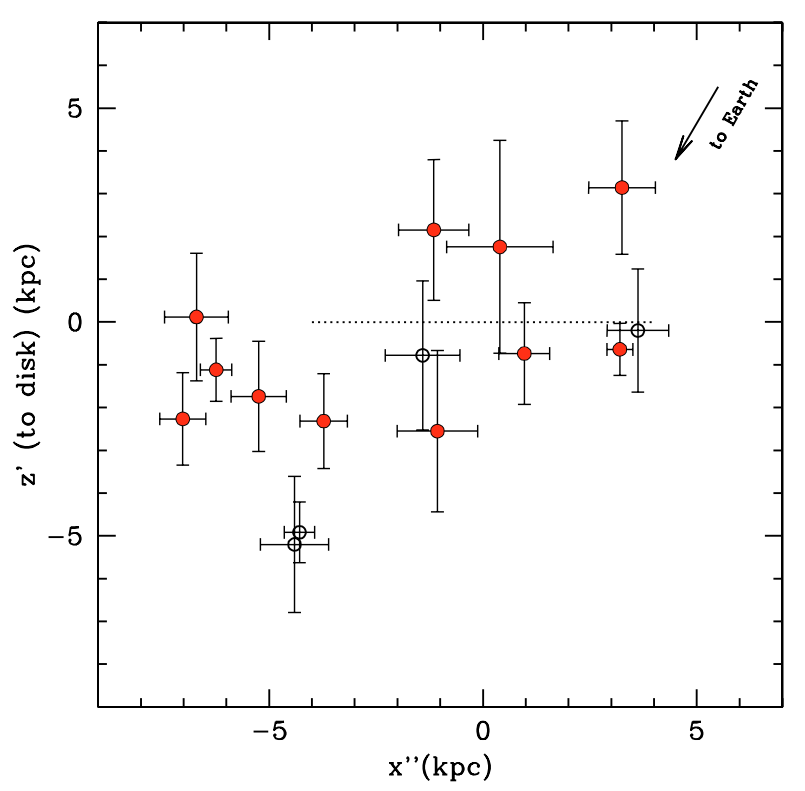

Fig. 35. Spatial distribution for the LMC clusters in a plane aligned to the LMC disk (indicated by the dotted line). The direction towards us is shown in the top-right corner.

figure is consistent with a clockwise rotation curve, with the extreme NE $\left(-x^{\prime}\right)$ or SW $\left(+x^{\prime}\right)$ clusters with the highest absolute values for the radial velocities. This rotation curve, combined with the derived inclination for the cluster distribution, suggests a (slightly) kinematically delayed structure compared to the bulk of stars in the LMC disk.

A plane with axes aligned with the LMC disk (the $z-x^{\prime}$ plane rotated clockwise by $i$ ) is presented in Fig. 35. Almost all clusters with the most accurate modelling are confined to a distance perpendicular to the disk plane lower than $3 \mathrm{kpc}$. 
Table A.1. Control experiments.

\begin{tabular}{|c|c|c|c|c|c|c|c|c|c|c|}
\hline \multirow[t]{2}{*}{ ID } & \multicolumn{4}{|c|}{ Input } & \multirow[t]{2}{*}{ Criterion } & \multicolumn{5}{|c|}{ Output } \\
\hline & $\log (\tau / \mathrm{yr})$ & $Z$ & $(m-M)_{0}$ & $E(B-V)$ & & $\log (\tau / \mathrm{yr})$ & $Z$ & $(m-M)_{0}$ & $E(B-V)$ & $N_{\text {best }}$ \\
\hline \multirow[t]{3}{*}{1} & 8.85 & 0.016 & 18.25 & 0.01 & MS & $8.81 \pm 0.03$ & $0.017 \pm 0.002$ & $18.30 \pm 0.09$ & $0.03 \pm 0.02$ & 83 \\
\hline & & & & & $\mathrm{RC}$ & $8.83 \pm 0.04$ & $0.016 \pm 0.002$ & $18.28 \pm 0.10$ & $0.02 \pm 0.02$ & 39 \\
\hline & & & & & MS\&RC & $8.84 \pm 0.02$ & $0.016 \pm 0.002$ & $18.26 \pm 0.07$ & $0.02 \pm 0.02$ & 21 \\
\hline \multirow[t]{3}{*}{2} & 9.05 & 0.004 & 18.35 & 0.04 & MS & $9.03 \pm 0.05$ & $0.005 \pm 0.001$ & $18.39 \pm 0.12$ & $0.04 \pm 0.03$ & 118 \\
\hline & & & & & $\mathrm{RC}$ & $9.02 \pm 0.06$ & $0.004 \pm 0.000$ & $18.42 \pm 0.09$ & $0.04 \pm 0.01$ & 25 \\
\hline & & & & & MS\&RC & $9.07 \pm 0.03$ & $0.004 \pm 0.000$ & $18.34 \pm 0.03$ & $0.04 \pm 0.01$ & 8 \\
\hline \multirow[t]{3}{*}{3} & 9.20 & 0.004 & 18.60 & 0.07 & MS & $9.20 \pm 0.06$ & $0.005 \pm 0.001$ & $18.56 \pm 0.12$ & $0.06 \pm 0.03$ & 244 \\
\hline & & & & & $\mathrm{RC}$ & $9.18 \pm 0.05$ & $0.005 \pm 0.001$ & $18.59 \pm 0.14$ & $0.05 \pm 0.03$ & 24 \\
\hline & & & & & MS\&RC & $9.20 \pm 0.04$ & $0.004 \pm 0.000$ & $18.62 \pm 0.10$ & $0.06 \pm 0.02$ & 12 \\
\hline \multirow[t]{3}{*}{4} & 9.45 & 0.008 & 18.25 & 0.07 & MS & $9.43 \pm 0.05$ & $0.008 \pm 0.002$ & $18.29 \pm 0.10$ & $0.07 \pm 0.03$ & 82 \\
\hline & & & & & $\mathrm{RC}$ & $9.45 \pm 0.10$ & $0.008 \pm 0.002$ & $18.25 \pm 0.06$ & $0.08 \pm 0.03$ & 48 \\
\hline & & & & & MS\&RC & $9.44 \pm 0.04$ & $0.008 \pm 0.001$ & $18.24 \pm 0.05$ & $0.08 \pm 0.02$ & 11 \\
\hline
\end{tabular}

\section{Summary and conclusions}

We analyzed HST/WFPC2 CMDs from 15 LMC populous clusters to determine the following physical parameters for each of them: age, metallicity, distance modulus and reddening. For each cluster, the observed MS fiducial line and RC position were simultaneously and statistically compared with the ones obtained from synthetic CMDs. The CMD models explored a regular grid in the parameter space consistent with previous determinations found in the literature. Control experiments were used to test our approach. Therefore, our determinations, based on photometrically homogeneous data, are self-consistent and done by an objective and robust method.

In general, the best models show a satisfactory fit to the data, reproducing well the MS fiducial line and RC features. Also, these models constrain well the physical parameters of each cluster, with typical uncertainties of 0.05 in $\log (\tau / \mathrm{yr}), 0.10 \mathrm{dex}$ in $[\mathrm{Fe} / \mathrm{H}], 0.05$ in $(m-M)_{0}$ and 0.02 in $E(B-V)$. These results are summarized in Table 2.

The AMR derived from our results has a lower spread in metallicity than the one compiled by Mackey \& Gilmore (2003) or the one recently determined by Leonardi \& Rose (2003) using integrated colors. We also recovered a mean $[\mathrm{Fe} / \mathrm{H}]$ of $\sim-0.50$, roughly $\sim 0.10$ more metal-poor than the Mackey \& Gilmore (2003) data and $\sim 0.20$ more metal-rich than Leonardi \& Rose (2003).

The metallicity values determined by us are in accordance with the ones from OSSH where more than one star per cluster was used to measure the $[\mathrm{Fe} / \mathrm{H}]$ cluster value. The uncertainties in our $[\mathrm{Fe} / \mathrm{H}]$ estimates are comparable with the ones obtained by OSSH, with the advantage that we recovered it based on a statistical method. Comparisons with previous metallicities determined using CMDs also reveal a good agreement, but those based on integrated light show some discrepant points.

In terms of age, the earlier determinations based on CMDs are in good agreement with ours for clusters older than $\log (\tau / \mathrm{yr}) \sim 9.0$. Below this limit, earlier results systematically recovered younger ages. The results from Leonardi \& Rose (2003) based on integrated light also show discrepancies for old clusters, in the sense that they obtained an older age than us.

In general, reddening and distance modulus have canonical values adopted in the previous studies, often not being consistently determined individually for each cluster. An exception is the $E(B-V)$ from McLaughlin \& van der Marel (2005), a study based on integrated colours, but their values have large uncertainties and do not allow a systematic comparison to our results. For NGC 1856, however, the cluster with the highest
$E(B-V)(\sim 0.21)$ value in our work, those authors also found their highest reddening value, in good agreement with us.

A consistent and expected relation involving reddening and distance modulus was found, in the sense that clusters with lower extinction tend to be in the foreground. The three-dimensional distribution of the clusters with the most reliable results seems to be roughly aligned with the LMC disk geometry, with a small difference of $\sim 8^{\circ}$ in the inclination, suggesting a (slightly) kinematically delayed structure for the system composed of the IACs in relation to the bulk of LMC disk stars. Although these clusters are restricted to a distance perpendicular to the disk lower than $3 \mathrm{kpc}$, they seem to be more scattered than the numerical predictions for the formation and evolution of intermediateage LMC clusters done by Bekki \& Chiba (2005). Therefore, the results of the three-dimensional distribution of the IACs in the LMC may be interpreted as an indication that these clusters were not formed in the LMC disk. Alternatively, they may have formed in the disk but been scattered away from it by interactions as they moved through the LMC potential.

We underline that the set of age and metallicities homogeneously derived here can be applied to calibrated light studies of distant galaxies. Since our results are based on the Padova models, it would also be very interesting to work with the same data to allow the intercomparison of predictions based on other stellar evolutionary models, like the $\mathrm{Y}^{2}$ (Yi et al. 2003), the Pisa (Castellani et al. 2003) and the Teramo (Pietrinferni et al. 2004) ones. Concerning these last models a quantitative result using synthetic CMDs to derive ages and reddenings of a small sample of LMC star clusters has been obtained by Raimondo et al. (2005) for a different purpose.

Acknowledgements. We thank Leo Girardi for kindly provide a thinner isochrone grid in metallicity. L.O.K. thanks Beatriz Barbuy for useful discussions and acknowledges FAPESP postdoctoral fellowship 05/01351-5.

\section{Appendix A: Control experiments}

This appendix shows some results of control experiments, used to test our approach to determine the physical parameters of a cluster. For each control experiment, a synthetic CMD with known (input) parameters is assumed as an "observed CMD" and compared with a regular model grid. Table A.1 presents the results (output) of a sample of such experiments, numbered from 1 to 4 , for a fixed $n=2$, and originally designed to quantify the formal uncertainties in the results for NGC 1831, NGC 1868, NGC 2173 and NGC 2121. As expected, the input parameters are recovered in the output for all experiments, attesting the applicability of our statistical tools. This table reveals that single 
criteria (MS or RC) have output parameters with higher uncertainties than the ones recovered when the both criteria are combined. These uncertainties are directly related to the number of models identified as best models ( $\left.N_{\text {best }}\right)$, as attested by the last column in this table.

\section{References}

Beasley, M. A., Hoyle, F., \& Sharples, R. 2002, MNRAS, 336

Bekki, K., \& Chiba, M. 2005, MNRAS, 356, 680

Bekki, K., Couch, W. J., Beasley, M. A., et al. 2004, ApJ, 610, L93

Bertelli, G., Nasi, E., Girardi, L., et al. 2003, AJ, 125, 770

Bica, E., Claria, J. J., Dottori, H., Santos., J. F., \& Piatti, A. E. 1996, ApJS, 102, 57

Bica, E., Geisler, D., Dottori, H., et al. 1998, AJ, 116, 723

Bica, E., Schmitt, H., Dutra, C., \& Oliveira, H. L. 1999, AJ, 117, 238

Brocato, E., Di Carlo, E., \& Menna, G. 2001, A\&A, 374, 523

Brocato, E., Castellani, V., Di Carlo, E., Raimondo, G., \& Walker, A. R. 2003, AJ, 125, 3111

Burstein, D., \& Heiles, C. 1982, AJ, 87, 1165

Castellani, V., Degl'Innocenti, S., Marconi, M., Prada Moroni, P. G., \& Sestito, P. 2003, A\&A, 404, 645

Clementini, G., Gratton, R., Bragaglia, A., et al. 2003, AJ, 125, 1309

Cole, A. A., Tolstoy, E., Gallagher, J. S., \& Smecker-Hane, T. A. 2005, AJ, 129, 1465

de Grijs, R., \& Anders, P. 2006, MNRAS, 366, 295

de Grijs, R., Gilmore, G., \& Mackey, A., et al. 2002, MNRAS, 337, 597

Dirsch, B., Richtler, T., Gieren, W. P., \& Hilker, M. 2000, A\&A, 360, 133

Dolphin, A. E. 2002, MNRAS, 332, 91

Elson, R. A., \& Fall, S. M. 1988, AJ, 96, 1383

Elson, R. A. W., Sigurdsson, S., Davies, M., Hurley, J., \& Gilmore, G. 1998, MNRAS, 300, 857

Gallart, C., Freedman, W. L., Aparicio, A., Bertelli, G., \& Chiosi, C. 1999, AJ, 118,2245

Gallart, C., Zoccali, M., Bertelli, G., et al. 2003, AJ, 125, 742

Geisler, D. 2006, oral contribution in Globular clusters, guides to galaxies, FONDAP/ESO Conf., Concepcion, Chile

Geisler, D., Bica, E., Dottori, H., et al. 1997, AJ, 114, 1920

Geisler, D., Piatti, A. E., Bica, E., \& Clariá, J. J. 2003, MNRAS, 341, 771

Girardi, L., Chiosi, C., Bertelli, G., \& Bressan, A. 1995, A\&A, 298, 87

Girardi, L., \& Bertelli, G. 1998, MNRAS, 300, 533
Girardi, L., Bertelli, G., Bressan, A., et al. 2002, A\&A, 391, 195

Girardi, L., Groenewegen, M. A. T., Hatziminaoglou, E., \& da Costa, L. 2005, A\&A, 436, 895

Goudfrooij, P., Gilmore, D., Kissler-Patig, M., \& Maraston, C. 2006, MNRAS, 369,697

Gouliermis, D., Keller, S. C., Kontizas, M., Kontizas, E., \& Bellas-Velidis, I. 2004, A\&A, 416, 137

Grocholski, A. J., Sarajedini, A., Olsen, K. A. G., \& Tiede, G. P. 2005 [arXiv:astro-ph/0506760]

Grocholski, A. J., Cole, A. A., Sarajedini, A., Geisler, D., \& Smith, V. V. 2006, AJ, 132, 1630

Hernandez, X., Valls-Gabaud, D., \& Gilmore, G., 1999, MNRAS, 304, 705

Javiel, S., Santiago, B., \& Kerber, L. 2005, A\&A, 431, 73

Leonardi, A. J., \& Rose, J. A. 2003, AJ, 126, 1811

Kerber, L. O., Santiago, B. X., Castro, R., \& Valls-Gabaud, D. 2002, A\&A, 390, 121

Kerber, L. O., \& Santiago, B. X 2005, A\&A, 435, 77

Kerber, L. O., \& Santiago, B. X. 2006, A\&A, 452, 155

Mackey, A. D., \& Gilmore, G. F. 2003, MNRAS, 338, 85

McLaughlin, D. E., \& van der Marel, R. P. 2005, AJ, 161, 304

Nikolaev, S., Drake, A. J., Keller, S. C., et al. 2004, ApJ, 601, 260

Olszewski, E. W., Schommer, R. A., Suntzeff, N. B., \& Harris, H. 1991, AJ, 101, 515 (OSSH)

Olszewski, E. W., Suntzeff, N. B., \& Mateo, M. 1996, ARA\&A, 34, 511

Piatti, A. E., Geisler, D., Bica, E., \& Clariá, J. J. 2003a, MNRAS, 343, 851

Piatti, A. E., Bica, E., Geisler, D., \& Clariá, J. J. 2003b, MNRAS, 344, 965

Pietrinferni, A., Cassisi, S., Salaris, M., \& Castelli, F. 2004, ApJ, 612, 168

Raimondo, G., Brocato, E., Cantiello, M., \& Capaccioli, M. 2005, AJ, 130, 2625

Rich, R. M., Shara, M. M., \& Zurek, D. 2001, AJ, 122, 842

Santos Jr., J. F. C., \& Piatti, A. E. 2004, A\&A, 428, 79

Santos Jr., J. F. C., Clariá, J. J., Ahumada, A. V., et al. 2006, A\&A, 448, 1023

Sarajedini, A. 1998, AJ, 116, 738

Sarajedini, A., Grocholski, A. J., Levine, J., \& Lada, E. 2002, AJ, 124, 2632

Schlegel, D. J., Finkbeiner, D. P., \& Davis, M. 1998, ApJ, 500, 525

Subramaniam, A. 2005, A\&A, 430, 421

Valls-Gabaud, D., \& Lastennet, E. 1999, Rev. Mex. Astron. Astrofis. Conf. Ser., 8,111

Westerlund, B. E. 1997, The Magellanic Clouds (Cambridge: Cambridge Univ. Press)

Woo, J.-H., Gallart, C., Demarque, P., et al. 2003, AJ, 125, 754

Yi, S. K., Kim, Y.-C., \& Demarque, P. 2003, ApJS, 144, 259

Zaritsky, D., Harris, J., Thompson, I. B., \& Grebel, E. K. 2004, AJ, 128, 1606 